\title{
Selected Lactobacillus Strains Inhibit Inflammation in LPS-induced RAW264.7 Macrophages by Suppressing the TLR4-Mediated NF-KB and MAPKs Activation
}

\author{
Jialu Shi \\ Northeast Agricultural University \\ Qinggang Xie \\ Heilongjiang Feihe Dairy Co., LTD \\ Huizhen Li \\ Northeast Agricultural University \\ Yingxue Yue \\ Northeast Agricultural University

\section{Smith Evivie} \\ University of Benin \\ $\mathrm{Na}$ Li \\ Northeast Agricultural University \\ Jiaqi Guan \\ Northeast Agricultural University \\ Bailiang Li ( $\sim 15846092362 @ 163 . c o m$ ) \\ Northeast Agricultural University \\ Guicheng Huo \\ Northeast Agricultural University
}

\section{Research Article}

Keywords: Lactobacillus Strains, Macrophages, MAPKs Activation, Pathogenic bacteria, oxidative stress, immunity disorder

Posted Date: December 17th, 2020

DOl: https://doi.org/10.21203/rs.3.rs-127575/v1

License: (9) This work is licensed under a Creative Commons Attribution 4.0 International License.

Read Full License 


\section{Abstract}

Pathogenic bacteria, oxidative stress, immunity disorder, and inflammation are involved in inflammatory bowel disease (IBD) development. Lactobacillus acidophilus KLDS 1.0901, Lactobacillus helveticus KLDS 1.8701, and Lactobacillus plantarum KLDS 1.0318 isolated from Chinese fermented dairy food with various functional characteristics, such as antibacterial, antioxidant, and immunomodulatory activity. Therefore, we selected three strains as a potential application for preventing IBD. However, the antiinflammatory properties of the tested strains are unknown. The current study evaluated the antiinflammatory potential of the tested strains using the LPS-induced RAW264.7 cells model. The results showed that all tested strains could inhibit iNOS and COX-2 expression, reducing NO and PGE2 production. And all tested strains markedly reduced the production and mRNA expression of proinflammatory cytokines (TNF-a, IL-1 $\beta$, and IL-6). Moreover, these results may be associated with inhibiting the translocation of NF-KB into nuclear and MAPKs activation. The study can provide evidence for further in vivo studies of Lactobacillus strains to prevent IBD.

\section{Introduction}

Inflammatory Bowel Diseases (IBD) has since been recognized as an emerging global public health ${ }^{1}$. Although the specific causative factors are not clear ${ }^{2}$, it is generally known that IBD is an inflammatory disease of the gastrointestinal tract, with some researchers speculating that the gut microbiota may play critical roles in its mitigation ${ }^{3}$. Also, several studies posit that the intestinal flora is not only involved in the occurrence of IBD but also could initiate the inflammatory cascade typical of IBD. When gut dysbiosis occurs as observed in IBD sufferers, the mucosal barrier function disappears, which results in the invasion of the mucosa region by pathogenic intestinal bacteria. Also, microbial antigens are activated, and the host's normal immune functions are disrupted, leading to oxidation and inflammation response ${ }^{4}$, 5 . Therefore, regulating the intestinal flora, inhibiting pathogenic bacteria, improving immunity status, reducing oxidative stress, and suppressing inflammation are essential for the prevention and treatment of IBD.

Inflammation is a defensive immune response to protect the body from exogenous stimuli. However, excessive or persistent inflammation can lead to various inflammation-related diseases ${ }^{6}$. Macrophages, derived from monocytes, play a crucial role in innate immunity by their unique phagocytosis function ${ }^{7}$. Lipopolysaccharide (LPS) comprises lipids and polysaccharides connected by a covalent bond and is the central part of the outer membrane of gram-negative bacteria. When pathogenic bacteria invasion occurs, the host and intestinal mucosa barrier are compromised, allowing LPS release into the bloodstream ${ }^{8}$. A combination of LPS, LPS binding protein (LBP), and CD14 forms the LPS-LBP-CD14 complex, which in turn binds to the Toll-like receptor 4 (TLR4)/ myeloid differentiation protein 2 (MD-2) on the mononuclearmacrophage membrane ${ }^{9}$. The TLR4 is stably expressed in cell membranes and binds to myeloid differentiation factor 88 (MyD88) through interactions with the Toll-interleukin-1 receptor (TIR) domains and activates the MyD88-dependent signaling pathway, responsible for downstream nuclear factor-kappa 
$\mathrm{B}$ (NF-KB) and mitogen-activated protein kinase (MAPKs) pathways ${ }^{10,11}$. The activation of NF-KB and MAPKs could influence inflammation, immune regulation, cell proliferation, and apoptosis. In the LPSstimulated murine RAW 264.7 cells, the expression of proinflammatory mediators and cytokines, including nitric oxide (NO), prostaglandin E2 (PGE2), tumor necrosis factor (TNF) -a, interleukin (IL) - $1 \beta$, IL6 , and IL-8, as well as other immune-related genes have been reported ${ }^{12}$. The RAW 264.7 cell model by LPS-inducing factors is widely used to study inflammatory response ${ }^{13}$.

Probiotics are living microorganisms that can provide beneficial effects to the host when administered in adequate amounts, such as regulation of gut microbiota, strengthening the intestinal barrier, and inhibiting intestinal diseases ${ }^{14}$. In recent years, Lactobacillus strains have been a probiotic focus group for the presentment and treatment of inflammations ${ }^{15,16}$. For instance, L. acidophilus SMC-S095 exerted protective effects against dextran sulfate sodium (DSS)-induced murine colitis. This strain downregulated the expression of IL-23 and transforming growth factor (TGF) - $\beta 1$ and the phosphorylation of signal transducer and activator of transcription (STAT) 3, resulting in decreased production levels of the proinflammatory cytokine IL-17 mediated by T helper cell (Th) 17 cells ${ }^{17}$. Furthermore, in LPS-stimulated RAW264.7 cells, the administration of $L$. helveticus NS8 can increase the production levels of IL-10 in peripheral blood mononuclear cells (PBMCs) in vivo ${ }^{18}$. Similarly, L. plantarum 21 significantly alleviated TNBS-induced colitis in rats by inhibiting TNF- $\alpha$ and IL- $1 \beta$ protein and mRNA expression in colon tissue, reducing the level of $\mathrm{NO}$, and increasing the content of reduced glutathione (GSH) and IL-10 protein and mRNA expression ${ }^{19}$.

L. acidophilus KLDS 1.0901, L. helveticus KLDS 1.8701, and L. plantarum KLDS 1.0318 were isolated from traditional fermented dairy food in Sinkiang (China) and identified using 16S rDNA sequence analysis. Our previous results showed that all tested strains exhibited high acid and bile salt-resistances and high cell adhesion. These characteristics implied that all tested strains could colonize the host intestine and confer probiotic properties. Furthermore, these strains displayed several health-promoting characteristics, such as antibacterial activity (L. acidophilus KLDS 1.0901) ${ }^{20}$, antioxidant activity ( $L$. helveticus KLDS 1.8701) ${ }^{21}$, and immunomodulatory activity (L. plantarum KLDS 1.0318) ${ }^{22}$.

As mentioned earlier, pathogenic bacteria, immunity disorder, oxidative stress, and inflammation are involved in IBD development. Therefore, we selected L. acidophilus KLDS 1.0901, L. helveticus KLDS 1.8701, and L. plantarum KLDS 1.0318 for potential IBD therapy applications. However, investigation of the anti-inflammatory properties of these three strains has not been carried out. The current study thus aimed to assess the potentials of $L$. acidophilus KLDS 1.0901, L. helveticus KLDS 1.8701, and $L$. plantarum KLDS 1.0318 in suppressing proinflammatory gene expressions and the related-signaling pathways (NF-KB and MAPK) activation in LPS-stimulated RAW 264.7 murine cell lines. We hope that this study will illustrate molecular evidence regarding these Lactobacillus strains in preventing IBD and provide the theoretical basis for future targeted in vivo experiments.

\section{Materials And Methods}


Bacterial strain and culture conditions. L. acidophilus KLDS 1.0901, L. helveticus KLDS 1.8701, and L. plantarum KLDS 1.0318 were isolated from traditional fermented dairy foods in Sinkiang Province (China) and preserved at the Northeast Agricultural University's (NEAU) Key Laboratory Dairy Science (KLDS) in Harbin, China. Strains were identified using the 16S rDNA sequence analysis and inoculated in improved Man Rogosa Sharpe (MRS) broth at $37^{\circ} \mathrm{C}$ for $18 \mathrm{~h}$ and were sub-cultured twice prior to the experiment. The bacterial cell pellets were harvested using centrifugation $(8000 \mathrm{rpm}, 10 \mathrm{~min})$ at $4^{\circ} \mathrm{C}$ and washed triple with sterile saline buffer $(\mathrm{pH}$ 7.4). The bacterial cell pellets were resuspended with high Dulbecco's modified Eagle's medium (DMEM) without antibiotics $(100 \mathrm{ug} / \mathrm{ml}$ streptomycin and $100 \mathrm{U} / \mathrm{ml}$ penicillin).

Cells Culture. The murine macrophage RAW264.7 cell line was purchased from the Chinese Academy of Sciences Cell Bank (Shanghai, China) and cultured in high DMEM medium including $10 \%$ fetal bovine serum (FBS) and $1 \%$ antibiotics at $37^{\circ} \mathrm{C}(5 \% \mathrm{CO} 2)$.

Cells viability. Murine RAW264.7 cells were cultivated in 96-well plates $(1.0 \times 105$ cells/well $)$ with $L$. acidophilus KLDS 1.0901, L. helveticus KLDS 1.8701, and L. plantarum KLDS 1.0318 suspensions at a multiplicity of infection (MOI) (ratio of bacteria number to epithelial cell number) of $10(1.0 \times$ $10^{6} \mathrm{CFU} /$ well $), 100\left(1.0 \times 10^{7} \mathrm{CFU} /\right.$ well $)$, and $1000\left(1.0 \times 10^{8} \mathrm{CFU} /\right.$ well $)$, respectively. After $24 \mathrm{~h}$ of incubation, DMEM with 10\% Cell Counting Kit-8 (CCK8) (APExBIO, USA) was added to each well, then in $37{ }^{\circ} \mathrm{C}(5 \% \mathrm{CO} 2)$ incubated for another $2 \mathrm{~h}$. The optical density (OD) was estimated at $450 \mathrm{~nm}$ using a microplate reader.

Nitric oxide (NO) Assay. Nitric oxide (NO) production in the culture supernatant was measured in LPSinduced RAW264.7 cells based on the Griess reaction, as previously described ${ }^{23}$. RAW264.7 cells were plated in 96-well plates $\left(1 \times 10^{5}\right.$ cells/well) and pre-incubated with L. acidophilus KLDS1.0901, L. helveticus KLDS 1.8701, and L. plantarum KLDS1.0318 at a MOI of $10\left(1.0 \times 10^{6} \mathrm{CFU} /\right.$ well $)$ for $1 \mathrm{~h}$, and then incubated with LPS $(1 \mathrm{ug} / \mathrm{ml})$ for $24 \mathrm{~h}$. Equal volumes of Griess reagent (Nanjing, China) were mixed with culture supernatants, and static for $10 \mathrm{~min}$ at room temperature. The absorbance was measured at $550 \mathrm{~nm}$ by an ultraviolet spectrometer.

Enzyme-Linked Immunosorbent Assay (ELISA). RAW264.7 cells were incubatied in 6-well plates $\left(1 \times 10^{6}\right.$ cells/well), and then treated with L. acidophilus KLDS 1.0901, L. helveticus KLDS 1.8701, and L. plantarum KLDS 1.0318 at an MOI of $10\left(1.0 \times 10^{7} \mathrm{CFU} /\right.$ well $)$ for $1 \mathrm{~h}$ to the stimulation with LPS (1 $\mathrm{ug} / \mathrm{ml}$ ) for another $24 \mathrm{~h}$. According to the ELISA kits manufacturer's instructions, the production levels of PGE2, TNF- $a$, IL-1 $\beta$, and IL- 6 were determined, respectively.

Real-time quantitative polymerase chain reaction (RT-qPCR). RAW264.7 cells were preincubated in 6-well plates $\left(1 \times 10^{6}\right.$ cells/well). Cells were treated with the L. acidophilus KLDS 1.0901, L. helveticus KLDS 1.8701 , and L. plantarum KLDS $1.0318\left(1.0 \times 10^{7} \mathrm{CFU} /\right.$ well $)$ for $1 \mathrm{~h}$ prior to the stimulation with LPS $(1 \mu \mathrm{g} / \mathrm{ml})$ for $24 \mathrm{~h}$. The total RNA of RAW264.7 cells was extracted using Total RNA Kit (Tiangen, China), and $2 \mu \mathrm{g}$ of the total RNA were reverse-transcribed to synthesize cDNA using a GoScript ${ }^{\mathrm{TM}}$ Reverse 
Transcription Mix (Promega, USA). PCR was amplificated and detected by GoTaq SYBR-Green qPCR Master Mix (Promega, USA) with 1 ug of CDNA. The relative expressions of target genes were analyzed using the $2^{-\triangle \Delta C t}$ calculation method. The gene primers were first mined from the NCBI database, then designed and synthesized by Sangon Biotech Co., Ltd (Shanghai, China) (Table 1.).

Table 1 Primers designed for this study

\begin{tabular}{|ll|}
\hline Genes & Primers \\
\hline INF-a & F: 5'-GCC TCT TCT CAT TCC TGC TTG TGG-3' \\
& R: 5'-GTG GTT TGT GAG TGT GAG GGT CTG-3' \\
& F: 5'-TCG CAG CAG CAC ATC AAC AAG AG-3' \\
& R: 5'-AGG TCC ACG GGA AAG ACA CAG G-3' \\
\hline IL-6 & F: 5'-AGA CAG CCA CCA CAC TGG AGA TAG-3' \\
& R: 5'-CCT GCC TCC TGT TGA TGT GAA GTC-3' \\
iNOS & F: 5'-ACT CAG CCA AGC CCT CAC CTA C-3' \\
R: 5'-TCC AAT CTC TGC CTA TCC GTC TCG-3' & F: 5'-GGT GCC TGG TCT GAT GAT GTA TGC-3' \\
& R: 5'-GGA TGC TCC TGC TTG AGT ATG TCG-3' \\
\hline TLR4 & F: 5'-CCG CTT TCA CCT CTG CCT TCA C-3' \\
& R: 5'-ACC ACA ATA ACC TTC CGG CTC TTG-3' \\
\hline B-actin & F: 5'- GGT TGT CTC CTG CGA CTT CA -3' \\
& R: 5'- TGG TCC AGG GTT TCT TAC TCC - 3' \\
\hline
\end{tabular}

Western blot analysis. RAW264.7 cells were planted in at a density of $1 \times 10^{6}$ cells/well and pretreated with L. acidophilus KLDS 1.0901, L. helveticus KLDS 1.8701, and L. plantarum KLDS 1.0318 (1.0 × $10^{7} \mathrm{CFU} /$ well) or special inhibitors for $1 \mathrm{~h}$ prior to the stimulation with LPS $(1 \mu \mathrm{g} / \mathrm{ml})$ for $20 \mathrm{~min}$ (NF-KB and MAPKs signal pathway) and $24 \mathrm{~h}$ (TLR4, COX-2 and iNOS). The cells were washed twice with ice-cold PBS and lysed with ice-cold radio-immunoprecipitation assay (RIPA) buffer (1 sodium dodecyl sulfate containing $1 \%$ phosphatase inhibitor, $0.1 \%$ protease inhibitor, and $0.5 \% 100 \mathrm{mM} \mathrm{PMSF}$ ). Total protein was extracted using the whole protein extraction kit (Invent Biotechnologies, USA), and concentrations required for analysis were quantified using the bicinchoninic acid (BCA, KeyGen, China) protein assay following the manufacturer's instructions. The total protein $(40 \mu \mathrm{g})$ of each sample was separated by 10\%-13\% sodium decyl sulfate-polyacrylamide gel electrophoresis (SDS-PAGE, KGP113, KeyGen, China) and transferred onto nitrocellulose/ polyvinylidene difluoride (NC/PVDF) membranes. The NC/PVDF membrane was blocked in buffer containing $5 \%$ skimmed milk with shaking for $2 \mathrm{~h}$, followed by incubation with primary antibodies that recognized p-38, p-p38, p65, JNK, TLR4, GAPDH (1:500; ImmunoWay, USA); ERK, p-ERK (1:1,000; Cell Signaling Technology, USA), IKB, p-IKB, p-JNK, $\beta$-actin 
(1:1,0000; Abcam, UK); COX2 (1:2000; Abcam, UK); iNOS (1:1000, Sangon Biotech, China) at $4^{\circ} \mathrm{C}$ overnight. The membrane was washed with TBST four times and probed with secondary antibodies (1:5000) for $1 \mathrm{~h}$ at room temperature. After washing thrice in TBST, the chemiluminescence detection system was enhanced with the ECL kit to display antibody-specific proteins.

Statistical analysis. All data were assessed using SPSS 23.0 software (SPSS Inc., Chicago, IL, USA) and expressed as mean \pm standard deviation (S.D.). The one-way analysis of variance (ANOVA) followed by Duncan's multiple range test was used to compare groups. In this study, $P$-values of $0.05,0.01$, and 0.001 were considered significant.

\section{Results}

The effect of the tested strains on cell viability. The effect of L. acidophilus KLDS 1.0901, L. helveticus KLDS 1.8701, and L. plantarum KLDS 1.0318 on cell viability was assessed by CCK8 assay. As shown in Fig. 1, the tested strains significantly inhibited cell viability within MOI of 100 and 1000 compared with that of the control group. These may have been induced by competition of nutrients between three strains and RAW264.7 macrophages. However, no significant differences in cell viability were observed between the control group and all tested strains-treated groups when the $\mathrm{MOI}$ is 10 . Given the above results, an $\mathrm{MOI}$ of 10 was applied during subsequent experiments.

Inhibition effect of the tested strains on inflammatory mediators. To evaluate the potential antiinflammatory property of the tested strains. The concentration of inflammatory mediators (NO and PGE2) and their relative mRNA (iNOS and COX-2) expressions were detected in LPS-stimulated RAW264.7 macrophages. Compared to the control group, the production of NO was increased in the LPS group (Fig. 2A). However, it was significantly reduced in the L. acidophilus KLDS $1.0901(P<0.001), L$. helveticus KLDS $1.8701(P<0.05)$, and L. plantarum KLDS $1.0318(P<0.001)$ group compared with that in the LPS group. This result was consistent with the changes of iNOS mRNA levels (Fig. 2C). As shown in Fig. 2B, the PGE2 level of the LPS group was markedly higher in contrast to that of the control group. However, treatment with L. acidophilus KLDS 1.0901, L. helveticus KLDS 1.8701, and L. plantarum KLDS 1.0318 decreased the production of PGE2 $(P<0.01, P<0.05, P<0.001)$. Furthermore, the expression level of COX-2 that regulated PGE2 production showed a similar trend (Fig. 2D). As aforementioned, all tested strains could decrease the concentration and gene expression of proinflammatory mediators. Furthermore, the L. plantarum KLDS 1.0318 had a more substantial inhibition effect in comparison to $L$. acidophilus KLDS 1.0901, followed by L. helveticus KLDS 1.8701.

Inhibition effect of the tested strains on inflammatory cytokines. In this study, we also evaluated the effect of these strains against proinflammatory cytokines by measuring the production of TNF- $a$, IL-1 $\beta$, IL6 and the expression of their mRNA. The TNF- $a$, IL-1 $\beta$, and IL- 6 concentration in the LPS group was significantly higher than that in the control group, implying that LPS treatment could increase the secretion of pro-inflammatory cytokines. However, the concentration of those proinflammatory cytokines was reduced markedly by all texted strains to the LPS group (Fig. 3A-3C). In terms of the transcription 
level of these cytokines, inhibition was also observed in Fig. 3D-F. Consistent with the above results, all tested strains could suppress TNF-a, IL-1 $\beta$, and IL- 6 mRNA expression to some extents $(P<0.001)$. In summary, the above results showed that all tested strains effectively inhibited the secretion of proinflammatory cytokines by down-regulating their gene transcription levels in LPS-induced RAW264.7 macrophages. Also, L. plantarum KLDS 1.0318 had the best anti-inflammatory effect.

Effect of the tested strains on TLR4 mRNA and protein expressions. To estimate the capacity of lactobacillus to inhibit LPS receptor expression, TLR4 mRNA transcription and protein expression levels were detected using RT-qPCR and western blot analysis, respectively. Compared with the control group and the LPS group, the relative expression of TLR4 mRNA increased from $1.35 \pm 0.27$ to $9.65 \pm 0.28$ by stimulating with LPS (Fig. 4A). However, compared to the LPS group, the TLR4 mRNA expression level was reduced to $5.79 \pm 0.21$ (L. acidophilus KLDS 1.0901), $7.17 \pm 0.40$ (L. helveticus KLDS 1.8701), and $4.89 \pm 0.34$ (L. plantarum KLDS 1.0318) following the treatment of the tested strains, respectively. As shown in Fig. 4B, LPS remarkably stimulated the protein expression of TLR4 compared with that of the control group, and this change was restored after all tested strains treatment. Compared to the LPS group, the TLR4 relative expressions with the tested strains were significantly down-regulated by $37.75 \%$ (L. acidophilus KLDS 1.0901), 28.32\% (L. helveticus KLDS 1.8701), and 58.70\% (L. plantarum KLDS 1.0318), respectively. These findings implied that all tested strains could significantly inhibit TLR4 mRNA and protein expression in the following order: L. plantarum KLDS $1.0318>$ L. acidophilus KLDS $1.0901>$ L. helveticus KLDS 1.8701 .

Effect of the tested strains on NF-KB activation. The critical role of NF-KB in inflammatory responses, which can stimulate the production of inflammatory factors. Therefore, we analyzed the inhibition effect of the tested strains on the NF-KB signaling pathway activation. As shown in Fig. 5A, after treatment with LPS, the relative expression $p$-IKB protein in the LPS group was markedly higher than that in the control group $(P<0.001)$. We also observed a significant reduction in the p65 protein expression level, and the trend of p65 protein expression in the nucleus was opposite to that of the cytosol (Fig. 5B), suggesting that the NF-KB p65 subunit is released from the complex and enters the nucleus. Compared to the LPS group, the $p$ - $\mid \kappa B$ relative expression with the tested strains pretreatment significantly declined by $4.05 \%$ ( $L$. acidophilus KLDS 1.0901), 8.20\% (L. helveticus KLDS 1.8701), and 22.43\% (L. plantarum KLDS 1.0318), respectively. And the nuclear protein expression of p65 decreased by $18.19 \%$ (L. acidophilus KLDS $1.0901), 16.88 \%$ (L. helveticus KLDS 1.8701), and 39.16\% (L. plantarum KLDS 1.0318) after the tested strains pretreatment compared with that of the LPS group, respectively. The above datas implied that all tested strains could inhibit the activation of the NF-KB signaling pathway by blocking the phosphorylation of IKB, thus reducing the transport of NF-KB p65 to the nucleus.

Effect of the tested strains on MAPKs activation. To determine whether the MAPKs signaling pathway has a dominant position of the anti-inflammatory potential of the tested strains against the inflammation response induced by LPS, the phosphorylation level of MAP kinases (p38, JNK, and ERK) in the RAW264.7 macrophages was measured. As shown in Fig. 6, the LPS group showed remarkable increase in the phosphorylation level of p38, JNK, and ERK compared with that of the control group $(P<0.001)$. 
However, all tested strains pretreatment restroed the changes in MAP kinase. The p-p38/p38 expression with the tested strains pretreated was significantly reduced by $12.97 \%$ (L. acidophilus KLDS 1.0901), $11.06 \%$ (L. helveticus KLDS 1.8701), and 27.14\% (L. plantarum KLDS 1.0318), respectively. Similarly, pERK/ERK protein level significantly down-regulated by $20.87 \%$ (L. acidophilus KLDS 1.0901 ), $23.05 \%$ (L. helveticus KLDS 1.8701), and $45.02 \%$ (L. plantarum KLDS 1.0318), respectively. Furthermore, the reduction of p-JNK/JNK protein expression $(24.39 \%, 7.03 \%$, and $16.86 \%)$ was found in L. acidophilus KLDS 1.0901, L. helveticus KLDS 1.8701, and L. plantarum KLDS 1.0318 group, respectively. The above results indicated that all texted strains could reduce the phosphorylation of p38, JNK, and ERK to suppress MAPKs signaling pathway activation.

Contrast KLDS 1.0318 and NF-KB /MAPKs inhibitors on inflammatory pathways. Based on the above results, NF-KB and MAPKs inhibitors were used to assess further the anti-inflammatory effect of $L$. plantarum KLDS 1.0318, which was most effective. As shown in Fig. 7, compared with the control group, the increase of iNOS and COX-2 protein expressions were found in the LPS group $(p<0.001)$. However, there was conspicuously decreased in the iNOS and COX-2 expression levels in L. plantarum KLDS 1.0318 group $(P<0.001)$, which showed the same trend with the BAY11-7082 (NF-kB inhibitor, 2.5

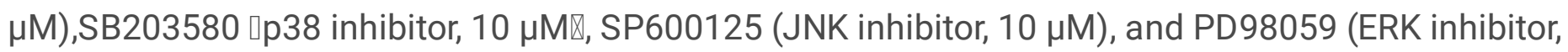
$10 \mu \mathrm{M}$ ) inhibitors group. In particular, the combination L. plantarum KLDS 1.0318 with specific inhibitors group showed more effective suppression, compared with L. plantarum KLDS 1.0318 or specific inhibitors alone. Furthermore, the iNOS level was more effectively suppressed by the combined treatment of L. plantarum KLDS 1.0318 with BAY11-708 or SB203580, compared with L. plantarum KLDS 1.0318 alone. Moreover, L. plantarum KLDS 1.0318 with PD98059 or BAY 11-708 showed a higher inhibitory effect than the L. plantarum KLDS 1.0318 group in terms of COX-2 expression. These findings indicated that L. plantarum KLDS 1.0318 exhibits synergistic inhibition when combined with the specific inhibitors.

\section{Discussion}

Inflammation responses can eliminate harmful external invasion or heal tissue injury. However, excessive inflammatory responses are closely related to the development of mild or chronic disease conditions. Therefore, reducing inflammation is a critical preventive and therapeutic strategy for controlling various diseases. Furthermore, due to the limitations and side effects of traditional anti-inflammatory drugs, it is essential to develop alternative measures that are effective and without side effects. For long-term oral administration and minimizing drug toxicity, there is currently no suitable food-grade products to treat inflammations. Many research works have shown that probiotics can improve infectious and inflammatory diseases and are widely used to prevent and treat IBD. Therefore, in our present study, the anti-inflammatory potential of L. acidophilus KLDS 1.0901, L. helveticus KLDS 1.8701, and L. plantarum KLDS 1.0318 was evaluated, and the associated cellular signaling pathways were investigated.

Drug cytotoxicity requires evaluation before development towards therapeutic applications. Therefore, we assessed the effect of L. acidophilus KLDS 1.0901, L. helveticus KLDS 1.8701, and L. plantarum KLDS 1.0318 on the viability of RAW 264.7 macrophages. All tested strains did not show cytotoxicity at the MOI 
of 10. Therefore, the tested strains $(\mathrm{MOI}=10)$ were further studied to illustrate the anti-inflammatory potential. NO and PGE2 are crucial biomarkers in the inflammatory response, modulated by iNOS and COX-2, respectively.

NO and PGE2 are crucial biomarkers in the inflammatory response, modulated by iNOS and COX-2, respectively ${ }^{24}$. Although the iNOS and COX-2 levels are not easily detected, LPS stimulated RAW264.7 cells to induce iNOS and COX-2 expression by activating NF-KB and MAPKs signal pathways to excessive NO production. At the inflammatory site, NO acts on vascular smooth muscle cells, which increases vascular permeability and accelerate the infiltration of inflammatory, pain mediators and monocytes into the inflammatory site. Besides, PGE2 is associated with fever, swelling, and pain perception ${ }^{25}$. Therefore, inhibiting the overexpression of iNOS and COX-2 is an essential strategy for the prevention and treatment of inflammatory diseases. In the current study, all tested strains significantly reduced the production of NO and PGE2 by inhibiting iNOS and COX-2 mRNA levels. These results are supported by many experimental pieces of evidence to validate claims that probiotics can ameliorate inflammation. The previous study demonstrated that Hwangryunhaedoktang weakly inhibited various inflammatory mediators (NO, PGE2, iNOS, and COX-2) induced by LPS. However, fermentation with lactobacilli significantly increased the inhibitory effect of the herbal compound, Hwangryunhaedoktang, on inflammatory mediator expression ${ }^{26}$. Especially among the tested strains, L. plantarum KLDS 1.0318 had the most potent inhibitory effect.

LPS-induced murine RAW264.7 cells produce several proinflammatory cytokines, including TNF-a, IL-1 $\beta$, and IL-6. Excessive release of proinflammatory cytokines can cause acute or chronic inflammatory diseases. For example, TNF-a is a key cytokine that stimulates the production of other cytokines in inflammatory responses and promotes the immune response process. ${ }^{27}$ However, compared to the LPS group, we discovered a remarkable reduction in the production and mRNA expression of proinflammatory cytokines (TNF-a, IL-1 $\beta$, and IL-6) in the L. acidophilus KLDS 1.0901, L. helveticus KLDS 1.8701, and L. plantarum KLDS 1.0318-treated groups. Consistent with these findings, L. plantarum $10 \mathrm{hk} 2$ isolated from fermented vegetables can reduce IL-1 $\beta$, IL-6, and TNF-a levels and increase the secretion of IL-10 in LPSinduced RAW 264.7 cells $^{28,29}$. Also, compared to the LPS group, we discovered a substantial decrease in the production and mRNA expression of proinflammatory cytokines (TNF- $a$, IL-1 $\beta$, and IL-6) with the treatment of L. acidophilus KLDS 1.0901, L. helveticus KLDS 1.8701, and L. plantarum KLDS 1.0318. In agreement with this observation, the $L$. plantarum $10 \mathrm{hk} 2$ strain also reduced the production of IL-1 $\beta$, IL- 6 , and TNF- $a$ and increased the secretion of IL-10 in LPS-induced RAW 264.7 cells ${ }^{30}$. The above results established the anti-inflammatory potential of L. acidophilus KLDS 1.0901, L. helveticus KLDS 1.8701, and L. plantarum KLDS 1.0318.

To further identify the possible mechanisms by which these strains confer the reported beneficial properties, we investigate whether TLR4-mediated NF-KB and MAPKs signaling pathways are associated with the anti-inflammatory ability L. acidophilus KLDS1.0901, L. helveticus KLDS1.8701, and L. plantarum KLDS1.0318. TLR4 is an integral receptor for LPS to activate NF-KB and MAPK pathways. NF- 
$K B$ signal plays a crucial role in gene expression in macrophages (Beinke, 2004). LPS induces degradation and ubiquitylation of IKB and NF-KB transfer into the nucleus; afterward, NF-KB regulates downstream gene transcription to promote the expression of proinflammatory cytokines (TNF-a, IL-6, and IL-1 $\beta$ ) and inflammatory mediators (NO and PGE2) ${ }^{31-34}$. In this study, the three strains studied showed significant inhibitory effects on the nuclear translocation of NF-KB. Similarly, Lactobacillus-fermented adlay-soymilk could suppress the production of PGE2 and NO and the synthesis of IL-1 $\beta$, IL- 6 , and TNF-a by down-regulated translocation of NF-KB p65 in the LPS-induced RAW164.7 cells $^{35}$.

The MAPKs are intracellular signaling molecules that regulate inflammatory responses through NF-KB activation and transcription. Currently, the MAPKs family includes three primary members, notably p38, JNK, and ERK regulating gene expression ${ }^{36,37}$. For example, COX is the rate-limiting enzyme for prostaglandin (PG) synthesis, and p38, JNK, and ERK are involved in the expression of COX-2. Furthermore, the MAPKs signaling pathway could regulate the secretion of cytokines through p38, JNK and ERK phosphorylation. The present study showed that treatment with all tested strains suppressed MAPKs signal pathway activation by reducing the phosphorylation of p38, JNK, and ERK factors. Previously, Lactobacillus brevis G-101 down-regulated TNBS-induced inflammatory signatures by suppressing the MAPKs phosphorylation in mice colitis ${ }^{38}$.

Generally, the maximum phosphorylation of LPS-induced MAP kinase is achieved within 30 min after LPS treatment and then rapidly decreased. Therefore, we measured the phosphorylation changes of MAP kinase after LPS stimulation for $20 \mathrm{~min}$. Also, the production of inflammatory mediators (NO and PGE2) and proinflammatory cytokines (TNF- $a$, IL-1 $\beta$, and IL-6) was measured for $24 \mathrm{~h}$. However, the data did not specifically show the change of NF-KB and MAP kinase activity in the late status of LPS-stimulated RAW 264.7 cells. Therefore, we aimed to determine the effect of LPS treatment on NF-KB and MAPKs signaling pathways in the late stage, using strategic co-administration of L. plantarum KLDS 1.0318 with specific inhibitors, thereby decreasing the protein level of the downstream target genes (iNOS and COX-2) ${ }^{31}$. The results showed that each combined treatment (L. plantarum KLDS $1.0318+$ a specific inhibitor) exhibited better inhibition on LPS- stimulated iNOS and COX-2 expressions. Specifically, LPS-induced iNOS expression was markedly suppressed by the combinations of $L$. plantarum KLDS 1.0318 with BAY 11708 and with SB203580. Moreover, LPS-stimulated COX-2 expression was significantly inhibited by $L$. plantarum KLDS 1.0318 with PD98059, and with BAY 11-708.

We investigated the anti-inflammatory potential of L. acidophilus KLDS 1.0901, L. helveticus KLDS 1.8701, and L. plantarum KLDS 1.0318 isolated from traditional dairy products in LPS-stimulated murine RAW264.7 cells. All tested strains could inhibit inflammatory mediators (NO and PGE2) and proinflammatory cytokines (TNF-a, IL-1 $\beta$, and IL-6) though down-regulating their relative gene expressions. This inhibitory function was related to reduced translocation of NF-KB into the nucleus and the phosphorylation of MAP kinase. This study provides that L. acidophilus KLDS 1.0901, L. helveticus KLDS 1.8701, and L. plantarum KLDS 1.0318 could be functional probiotics to prevent inflammationassociated disorders. 


\section{Declarations}

Acknowledgments

Grants from the National Key Research and Development Program of China (No. 2017YFD0400303) and "Hundred, Thousand and Ten Thousand" Science and Technology Major Special Project of Heilongjiang Province: Dairy Products and Meat Processing (No. 2019ZX07801), and Academic Backbone Plan of Northeast Agricultural University (No. 19YJXG10) financially supported this research.

Author contributions statement

B.L. and G.H. planned and supervised the experiments; J.S. and Q.X. carried out the experiments; J.S., H.L., Y.Y. and S.E. analyzed the data; J.S., N.L. and J.G. prepared Figures; all authors review the manuscript; all authors have read and agreed to the published version of the manuscript.

Conflict of Interest

The authors declare that they have no conflicts of interest.

\section{References}

1. G. Bouma and W. Strober, The immunological and genetic basis of inflammatory bowel disease, Nature Reviews Immunology, 2003, 3, 521-533.

2. Y. Peng, Y. Yan, P. Wan, D. Chen, Y. Ding, L. Ran, J. Mi, L. Lu, Z. Zhang, X. Li, X. Zeng and Y. Cao, Gut microbiota modulation and anti-inflammatory properties of anthocyanins from the fruits of Lycium ruthenicum Murray in dextran sodium sulfate-induced colitis in mice, Free Radical Biology and Medicine, 2019, 136, 96-108.

3. R. J. Farrell and J. T. LaMont, Microbial factors in inflammatory bowel disease, Gastroenterology Clinics of North America, 2002, 31, 41-62.

4. A. Nishida, R. Inoue, O. Inatomi, S. Bamba, Y. Naito and A. Andoh, Gut microbiota in the pathogenesis of inflammatory bowel disease, Clinical Journal of Gastroenterology, 2018, 11, 1-10.

5. S. Schreiber, R. P. MacDermott, A. Raedler, R. Pinnau, M. J. Bertovich and G. S. Nash, Increased activation of isolated intestinal lamina propria mononuclear cells in inflammatory bowel disease, Gastroenterology, 1991, 101, 1020-1030.

6. H. N. Lee, H. K. Na and Y. J. Surh, Resolution of inflammation as a novel chemopreventive strategy, Semin Immunopathol, 2013, 35, 151-161.

7. H. W. Ryu, S. U. Lee, S. Lee, H. H. Song, T. H. Son, Y. U. Kim, H. J. Yuk, H. Ro, C. K. Lee, S. T. Hong and S. R. Oh, 3-Methoxy-catalposide inhibits inflammatory effects in lipopolysaccharide-stimulated RAW264.7 macrophages, Cytokine, 2017, 91, 57-64.

8. X. Guo and J. Chen, The protective effects of saxagliptin against lipopolysaccharide (LPS)-induced inflammation and damage in human dental pulp cells, Artificial Cells Nanomedicine and 
Biotechnology 2019, 47, 1288-1294.

9. Y. C. Lu, W. C. Yeh and P. S. J. C. Ohashi, LPS/TLR4 signal transduction pathway, 2008, 42, 0-151.

10. P. R. Rauta, M. Samanta, H. R. Dash, B. Nayak and S. Das, Toll-like receptors (TLRs) in aquatic animals: Signaling pathways, expressions and immune responses, Immunology Letters, 2014, 158, 14-24.

11. S. F. Yang, T. F. Zhuang, Y. M. Si, K. Y. Qi and J. Zhao, Coriolus versicolor mushroom polysaccharides exert immunoregulatory effects on mouse $B$ cells via membrane Ig and TLR-4 to activate the MAPK and NF-KB signaling pathways, Molecular Immunology, 2015, 64, 144-151.

12. T. Lawrence, D. A. Willoughby and D. W. Gilroy, Anti-inflammatory lipid mediators and insights into the resolution of inflammation, Nature Reviews Immunology, 2002, 2, 787-795.

13. Y. Lu, S. J. Suh, C. H. Kwak, K. M. Kwon, C. S. Seo, Y. Li, Y. Jin, X. Li, S. L. Hwang and O. Kwon, Saucerneol F, a new lignan, inhibits iNOS expression via MAPKs, NF-KB and AP-1 inactivation in LPSinduced RAW264.7 cells, International Immunopharmacology, 2012, 12, 0-181.

14. S. L. Gorbach, Probiotics and gastrointestinal health, American Journal of Gastroenterology, 2000, 95.

15. R. N. Fedorak, P. Gionchetti, M. Campieri, K. Madsen, K. Isaacs, C. Desimone and B. Sartor, VSL3 Probiotic mixture induces remission in patients with active ulcerative colitis, Gastroenterology, 2003, 124, A377-A377.

16. L. I. Xin, M. K. Chen, J. L. Jin, H. Y. Zhou and Z. Tian, Clinical Effect of Probiotics on the Ulcerative Colitis and Its Mechanisms, Progress in Modern Biomedicine, 2016, 16, 5361-5363.

17. L. Chen, Y. Zou, J. Peng, F. Lu, Y. Yin, F. Li and J. Yang, Lactobacillus acidophilus suppresses colitisassociated activation of the IL-23/Th17 axis, Journal of Immunology Research, 2015, $2015,909514$.

18. J. Rong, H. Zheng, M. Liu, X. Hu, T. Wang, X. Zhang, F. Jin and L. Wang, Probiotic and antiinflammatory attributes of an isolate Lactobacillus helveticus NS8 from Mongolian fermented koumiss, BMC Microbiol, 2015, 15, 196.

19. C. S. V. S. Kumar, K. K. Reddy, A. G. Reddy, A. Vinoth, S. R. C. Ch, G. Boobalan and G. S. Rao, Protective effect of Lactobacillus plantarum 21, a probiotic on trinitrobenzenesulfonic acid-induced ulcerative colitis in rats, International Immunopharmacology, 2015, 25, 504-510.

20. J. C. Du, L. Fei, B. L. Li, B. Xin, S. E. Evivie, X. Min, X. Y. Ding and G. C. Huo, In Vitro Assessment of Probiotic Potential of Lactobacillus acidophilus and Antagonistic Activity Against Escherichia coil 0157:H7, Journal of Northeast Agricultural University, 2017, 024, 59-69.

21. B. Li, P. Du, E. E. Smith, S. Wang, Y. Jiao, L. Guo, G. Huo and F. Liu, In vitro and in vivo evaluation of an exopolysaccharide produced by Lactobacillus helveticus KLDS1.8701 for the alleviative effect on oxidative stress, Food \& Function, 2019, 10, 1707-1717.

22. Y. Meng, J. Wang, Z. Wang, G. Zhang and L. Liu, Lactobacillus plantarum KLDS1.0318 Ameliorates Impaired Intestinal Immunity and Metabolic Disorders in Cyclophosphamide-Treated Mice, Frontiers in Microbiology, 2019, 10. 
23. Ji-Won, Park, Ok-Kyoung, Kwon, Jung-Hee, Kim, Sei-Ryang, Oh, Jae-Hong and J.-H. J. I. j. o. m. medicine, Rhododendron album Blume inhibits iNOS and COX-2 expression in LPS-stimulated RAW264.7 cells through the downregulation of NF-kB signaling, 2015.

24. L. J. F. Sautebin, Prostaglandins and nitric oxide as molecular targets for anti-inflammatory therapy, Fitoterapia, 2000, 71 S48-57.

25. E. Degagne, D. M. Grbic, A. A. Dupuis, E. G. Lavoie, C. Langlois, N. Jain, G. A. Weisman, J. Sevigny and F. P. Gendron, P2Y2 Receptor Transcription Is Increased by NF-KB and Stimulates Cyclooxygenase-2 Expression and PGE2 Released by Intestinal Epithelial Cells, Journal of Immunology Research, 2009, 183, 4521-4529.

26. B. H. Han, J. L. Yun, J. J. Yoon, E. S. Choi, S. Namgung, J. J. Xian, H. J. Da, D. G. Kang and H. S. Lee, Hwangryunhaedoktang exerts anti-inflammation on LPS-induced NO production by suppressing MAPK and NF-KB activation in RAW264.7 rmacrophages, Journal of Integrative Medicine, 2017, 015, 326-336.

27. J. Shou, X. Kong, X. Wang, Y. Tang, C. Wang, M. Wang, L. Zhang, Y. Liu, C. Fei and F. Xue, Tizoxanide Inhibits Inflammation in LPS-Activated RAW264.7 Macrophages via the Suppression of NF-KB and MAPK Activation, Inflammation, 2019, 42, 1336-1349.

28. S. Cuzzocrea, L. Sautebin, G. D. Sarro, G. Costantino and C. Thiemermann, Role of IL-6 in the pleurisy and lung injury caused by carageenan, Journal of Immunology Research, 1999, 163, 5094-5104.

29. U. P. Singh, N. P. Singh, E. A. Murphy, R. L. Price, R. Fayad, M. Nagarkatti and P. S. J. C. Nagarkatti, Chemokine and cytokine levels in inflammatory bowel disease patients, 2016.

30. H. Chon, B. Choi, G. Jeong, E. Lee and S. Lee, Suppression of proinflammatory cytokine production by specific metabolites of Lactobacillus plantarum 10hk2 via inhibiting NF-KB and p38 MAPK expressions, Comparative Immunology, Microbiology and Infectious Diseases, 2010, 33, e41-e49.

31. W. Dan-Dan, P. Wen-Juan, M. Shomaila, C. Xiao-Du and C. Yan, Polysaccharide isolated from Sarcodon aspratus induces RAW264.7 activity via TLR4-mediated NF-KB and MAPK signaling pathways, International Journal of Biological Macromolecules, 2018, DOI:

10.1016/j.jbiomac.2018.08.147, S0141-8130(0118)33294-X

32. S. J. Wu, Y. W. Chen, C. Y. Wang, Y. T. J. I. J. o. F. S. Shyu and Technology, Anti-inflammatory properties of high pressure-assisted extracts of Grifola frondosa in lipopolysaccharide-activated RAW 264.7 macrophages, 2017, 52, 671-678.

33. X. Xu, P. Yin, C. Wan, X. Chong and J. Xu, Punicalagin inhibits inflammation in LPS-induced RAW264.7 macrophages via the suppression of TLR4-mediated MAPKs and NF-KB activation, Inflammation, 2014, 37.

34. Y. XUE, Y. WANG, D.-c. FENG, B.-g. XIAO and L.-y. X. J. A. P. Sinica, Tetrandrine suppresses lipopolysaccharide-induced microglial activation by inhibiting NF-kB pathway, 2008.

35. S. J. Wu, J. Y. Fang, C. C. Ng, C. Y. Wang and Y. T. Shyu, Anti-inflammatory activity of Lactobacillusfermented adlay-soymilk in LPS-induced macrophages through suppression of NF-KB pathways, Food Research International, 2013, 52, 262-268. 
36. S. F. I. P., A. S. K. K., S. K. W., L. W. Woo, K. Hyun-Soo, R. P., G. U. K. D. S. S. and J. You-Jin, Antioxidant and anti-inflammatory functionality of ten Sri Lankan seaweed extracts obtained by carbohydrase assisted extraction, Food Science Biotechnology, 2018, 27.

37. Wei, Zhang, Hui, Tu and L. J. C. research, MAPK signal pathways in the regulation of cell proliferation in mammalian cells, 2002.

38. S. E. Jang, S. R. Hyam, M. J. Han, S. Y. Kim, B. G. Lee and D. H. Kim, Lactobacillus brevis G-101 ameliorates colitis in mice by inhibiting NF-KB, MAPK and AKT pathways and by polarizing M1 macrophages to M2-like macrophages. , Journal of Applied Microbiology, 2013, 115, 888-896.

\section{Figures}

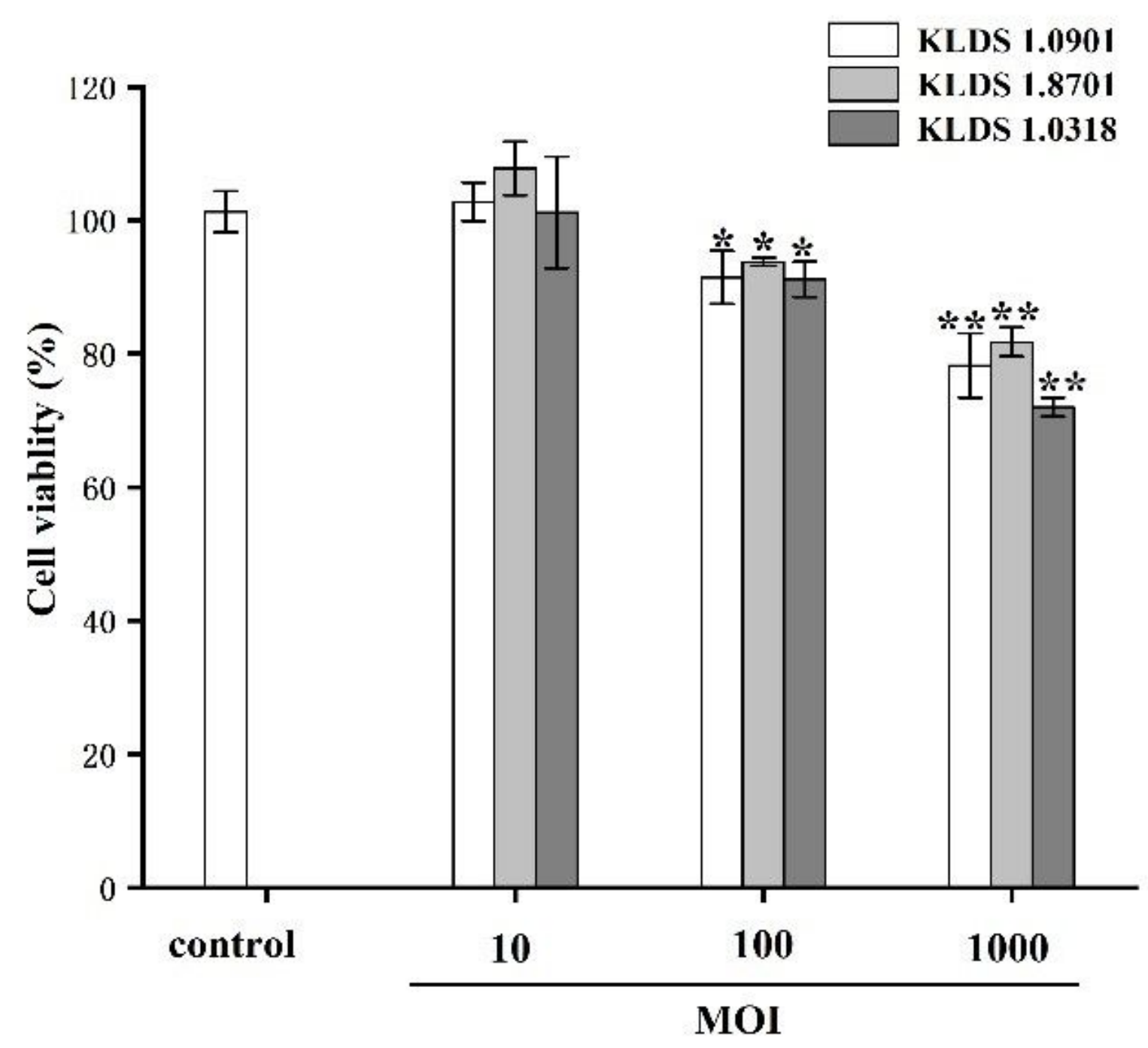

Figure 1

Effects of the tested strains at different MOI on the cell viability by the CCK8 assay. Data are expressed as means $\pm S D(n=6) .{ }^{*} P<0.05$ and $* * P<0.01$ vs. the control group. 


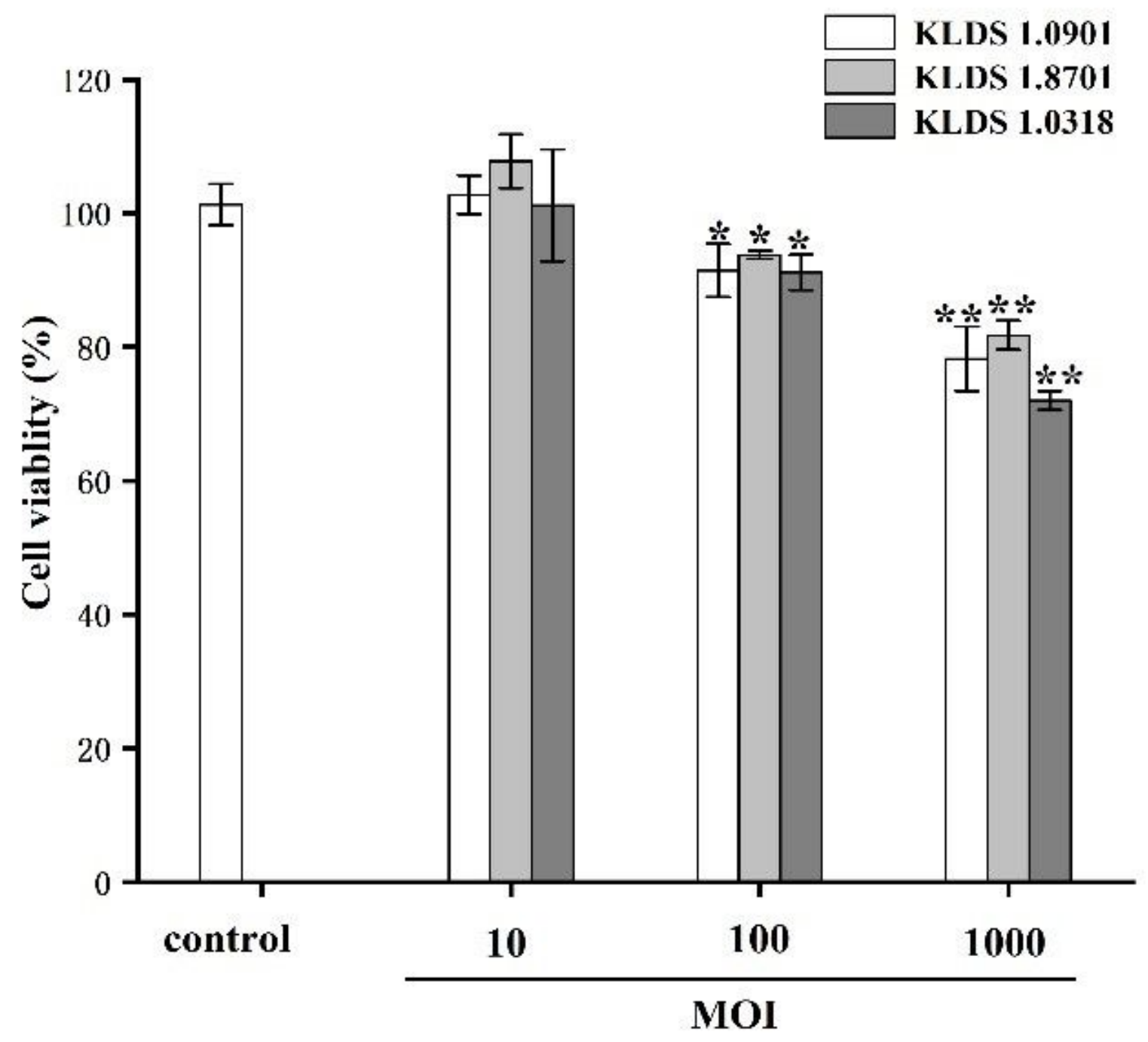

Figure 1

Effects of the tested strains at different MOI on the cell viability by the CCK8 assay. Data are expressed as means $\pm S D(n=6) .{ }^{*}<0.05$ and $* * P<0.01$ vs. the control group. 

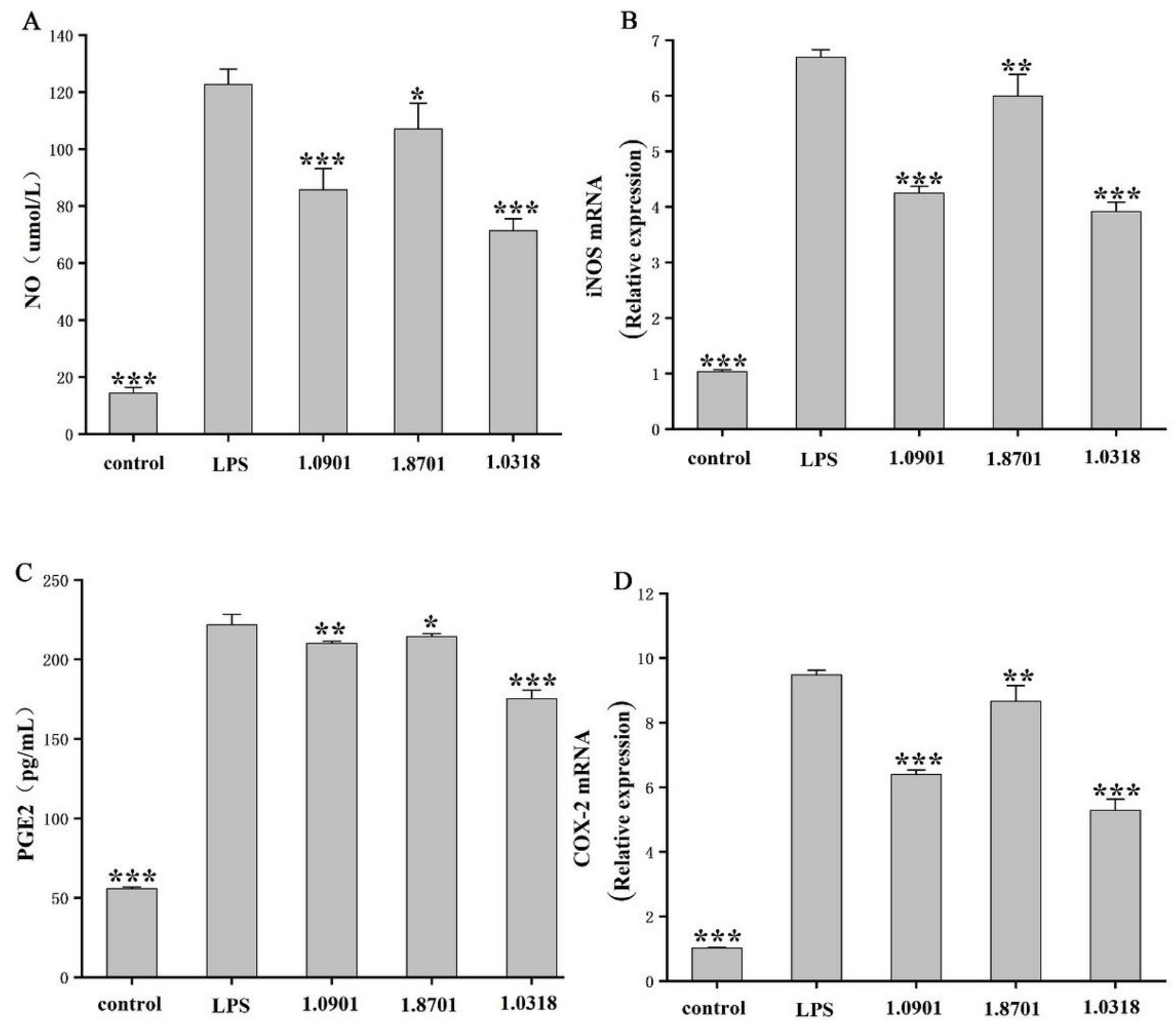

Figure 2

Effects of the tested strains on NO (A), PGE2 (B) production and iNOS (C), COX-2 (D) mRNA expression in LPS-induced RAW 264.7 cells. Cells were pretreated with L. acidophilus KLDS1.0901, L. helveticus KLDS1.8701 and L. plantarum KLDS1.0318 for $1 \mathrm{~h}$ and then stimulated with LPS for $12 \mathrm{~h}$. Data are expressed as means $\pm S D(n=3) .{ }^{*} P<0.05, * \star P<0.01$ and ${ }^{\star \star \star} P<0.001$ vs. the LPS group. 

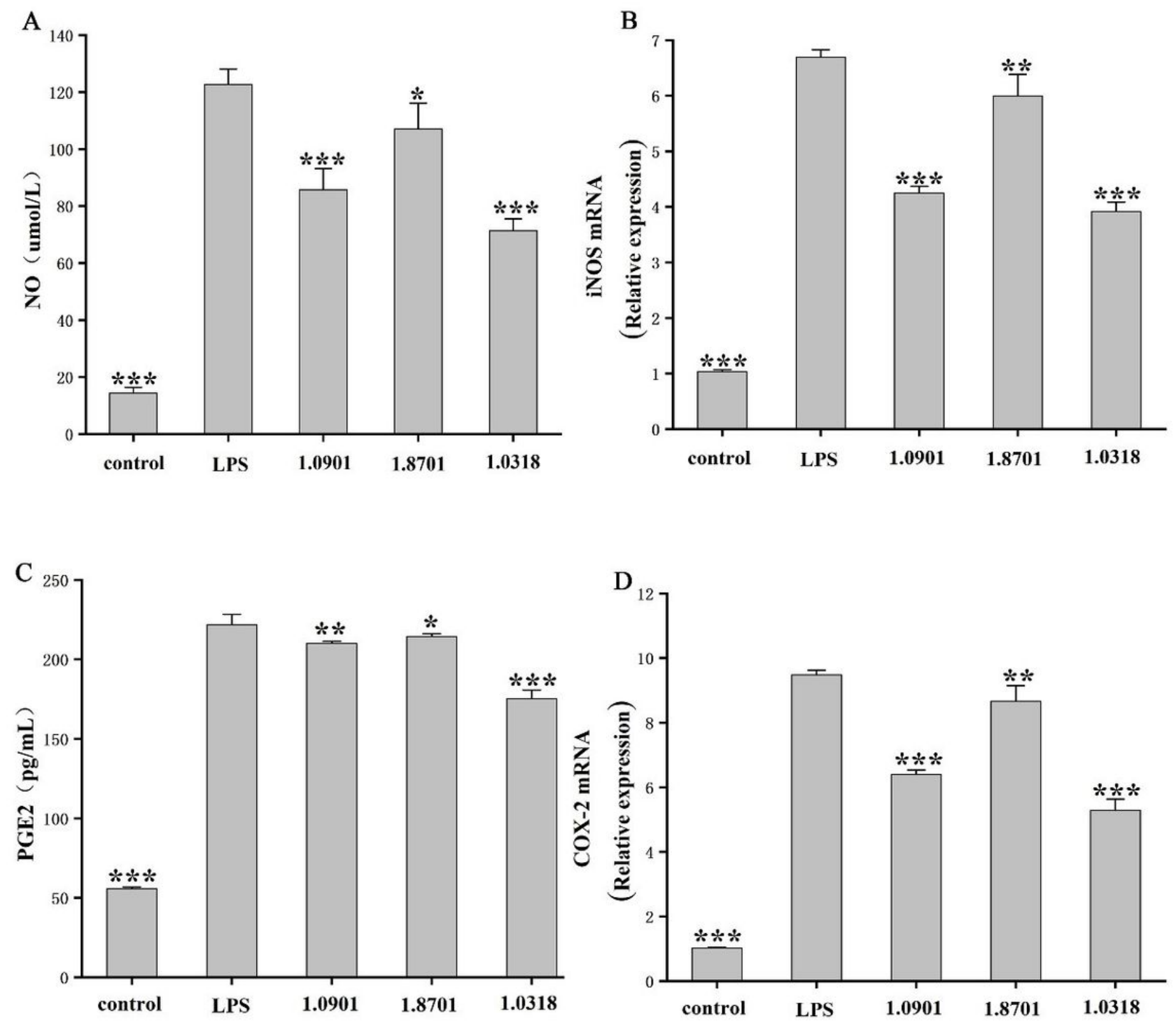

Figure 2

Effects of the tested strains on NO (A), PGE2 (B) production and iNOS (C), COX-2 (D) mRNA expression in LPS-induced RAW 264.7 cells. Cells were pretreated with L. acidophilus KLDS1.0901, L. helveticus KLDS1.8701 and L. plantarum KLDS1.0318 for $1 \mathrm{~h}$ and then stimulated with LPS for $12 \mathrm{~h}$. Data are expressed as means $\pm S D(n=3) .{ }^{*} P<0.05, * \star P<0.01$ and ${ }^{\star \star \star} P<0.001$ vs. the LPS group. 

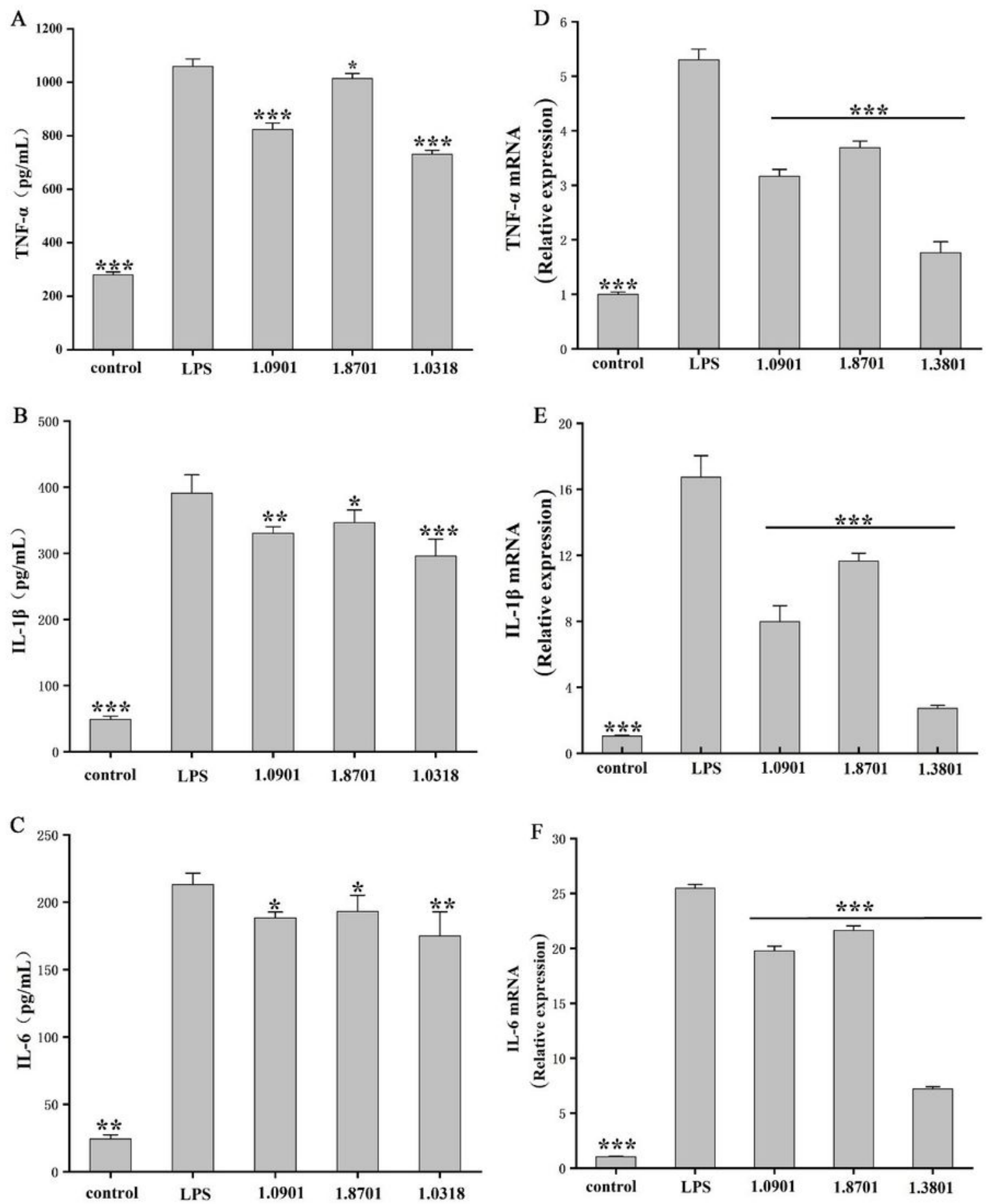

\section{Figure 3}

Effects of the tested strains on production and gene expression of pro-inflammatory cytokines. Cells were with L. acidophilus KLDS1.0901, L. helveticus KLDS1.8701 and L. plantarum KLDS1.0318 for $1 \mathrm{~h}$. TNF-a (A), IL-1 $\beta$ (B) and IL-6 (C) production and TNF- $\alpha(D), I L-1 \beta(E)$ and IL-6 (F) mRNA expression were detected by ELISA and RT-qPCR after LPS stimulated for $12 \mathrm{~h}$. Data are expressed as means $\pm S D(n=3)$. $P<$ 0.05 , ** $\mathrm{P}<0.01$ and $* * * \mathrm{P}<0.001$ vs. the LPS group. 

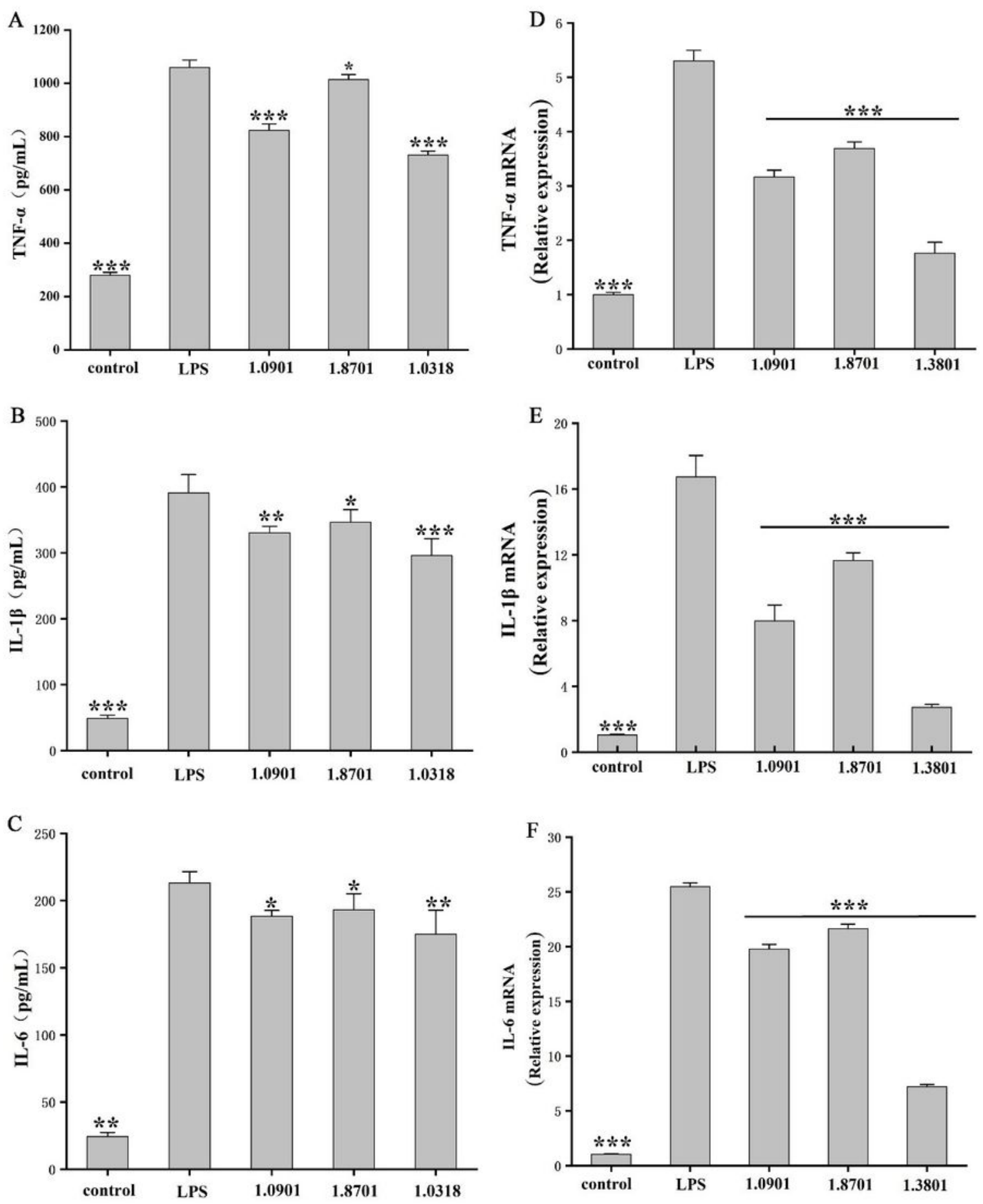

\section{Figure 3}

Effects of the tested strains on production and gene expression of pro-inflammatory cytokines. Cells were with L. acidophilus KLDS1.0901, L. helveticus KLDS1.8701 and L. plantarum KLDS1.0318 for $1 \mathrm{~h}$. TNF-a (A), IL-1 $\beta$ (B) and IL-6 (C) production and TNF- $\alpha(D), I L-1 \beta(E)$ and IL-6 (F) mRNA expression were detected by ELISA and RT-qPCR after LPS stimulated for $12 \mathrm{~h}$. Data are expressed as means $\pm S D(n=3)$. $P<$ 0.05 , ** $\mathrm{P}<0.01$ and $* * * \mathrm{P}<0.001$ vs. the LPS group. 

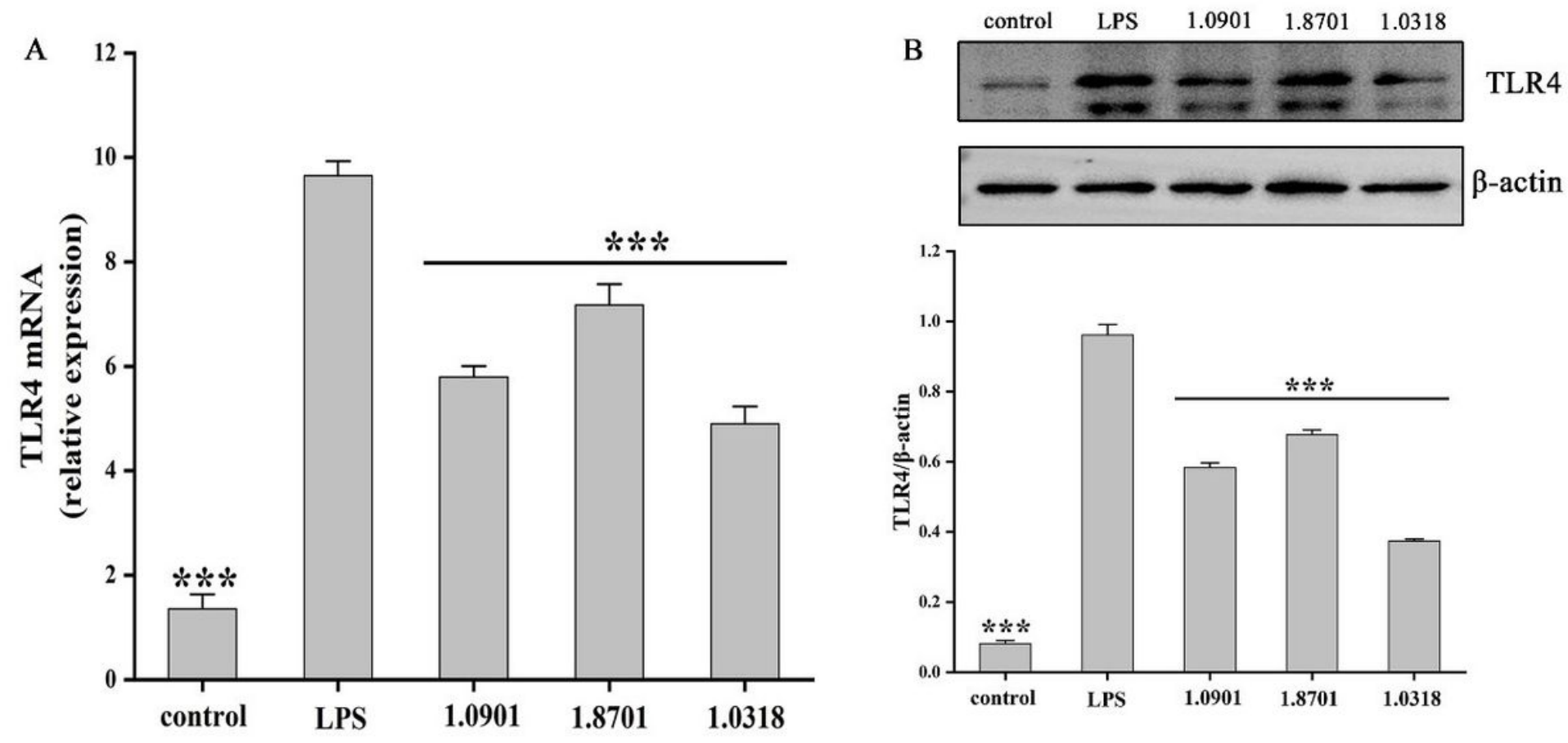

Figure 4

Effects of the tested strains on mRNA and protein relative expression of TLR4. Cells were with L. acidophilus KLDS1.0901, L. helveticus KLDS1.8701 and L. plantarum KLDS1.0318 for $1 \mathrm{~h}$. TLR4 mRNA (A) and protein (B) were determined by RT-qPCR and western blot analysis after LPS stimulated for $12 \mathrm{~h}$. Data are expressed as means $\pm S D(n=3) .{ }^{*} P<0.05$, $* * P<0.01$ and $* \star \star P<0.001$ vs. the LPS group.

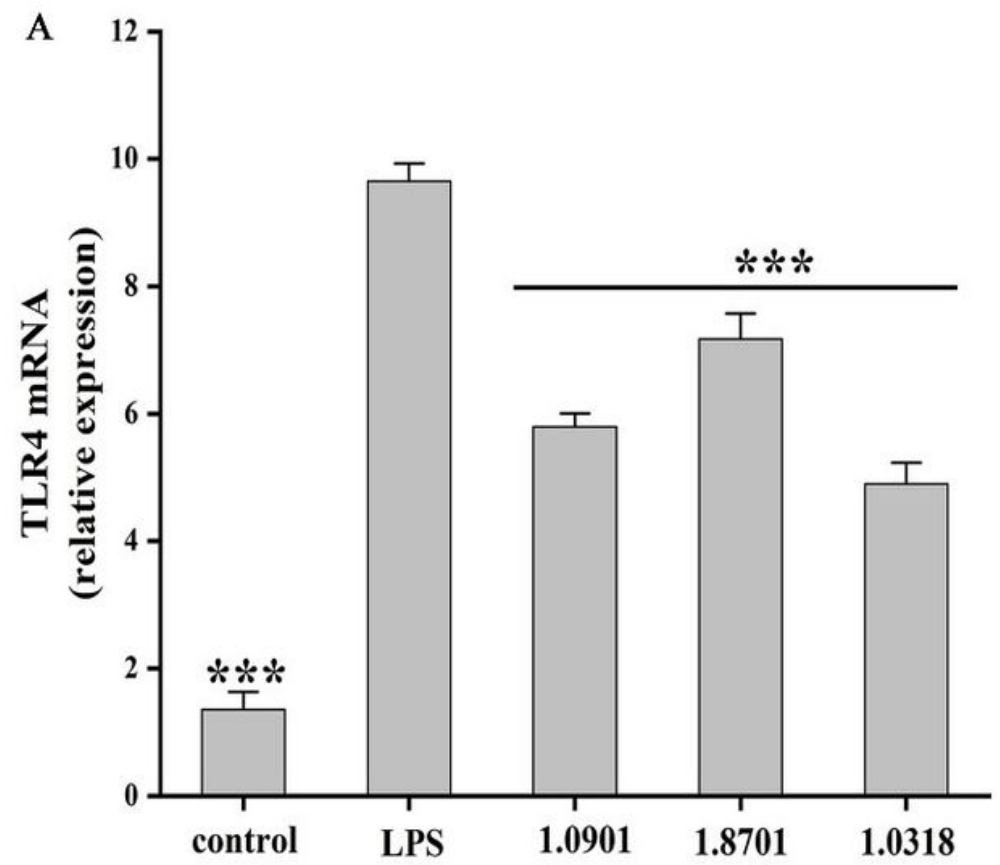

B

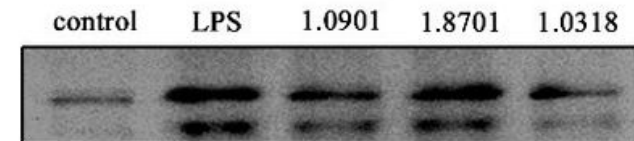

TLR4

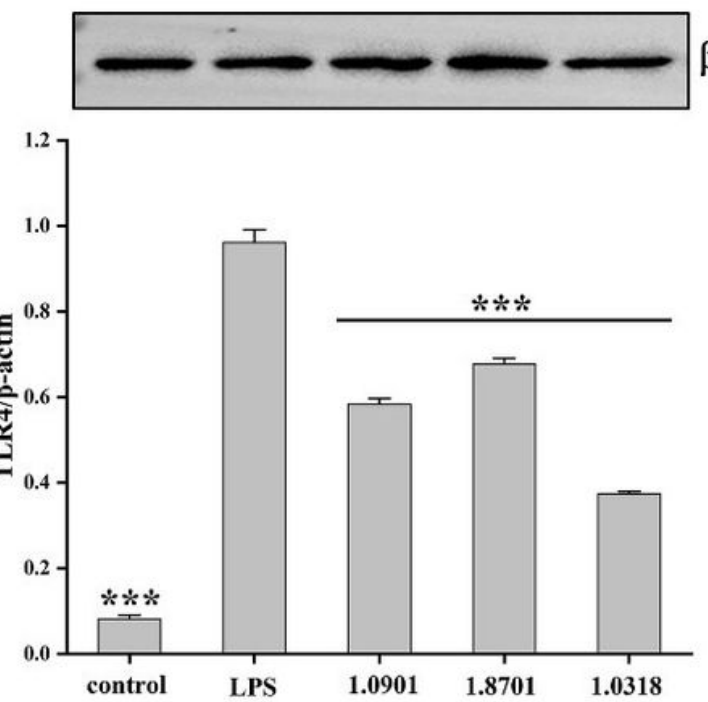


Figure 4

Effects of the tested strains on mRNA and protein relative expression of TLR4. Cells were with L. acidophilus KLDS1.0901, L. helveticus KLDS1.8701 and L. plantarum KLDS1.0318 for $1 \mathrm{~h}$. TLR4 mRNA (A) and protein (B) were determined by RT-qPCR and western blot analysis after LPS stimulated for $12 \mathrm{~h}$. Data are expressed as means $\pm S D(n=3)$. ${ }^{*} P<0.05$, $* \star P<0.01$ and $* \star \star P<0.001$ vs. the LPS group.

A

control LPS $1.09011 .8701 \quad 1.0318$

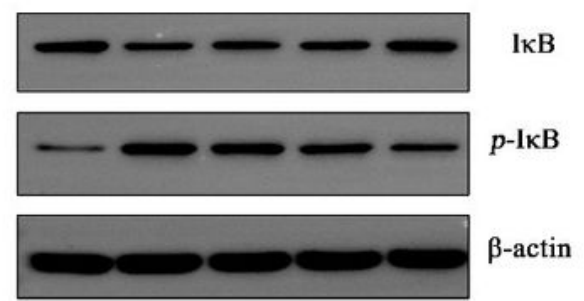

\section{Cytosol}

B

control LPS 1.09011 .87011 .0318
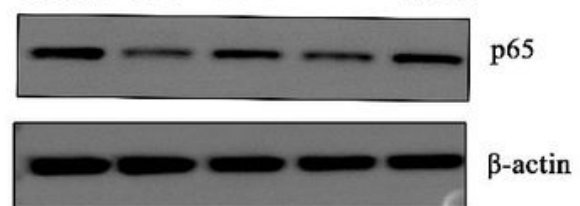

Nucleus

control LPS $1.09011 .8701 \quad 1.0318$
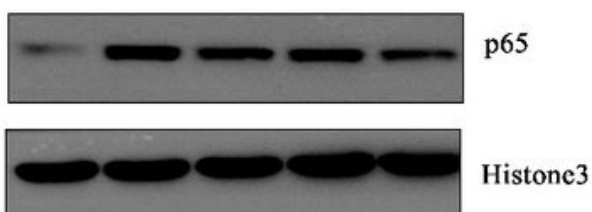
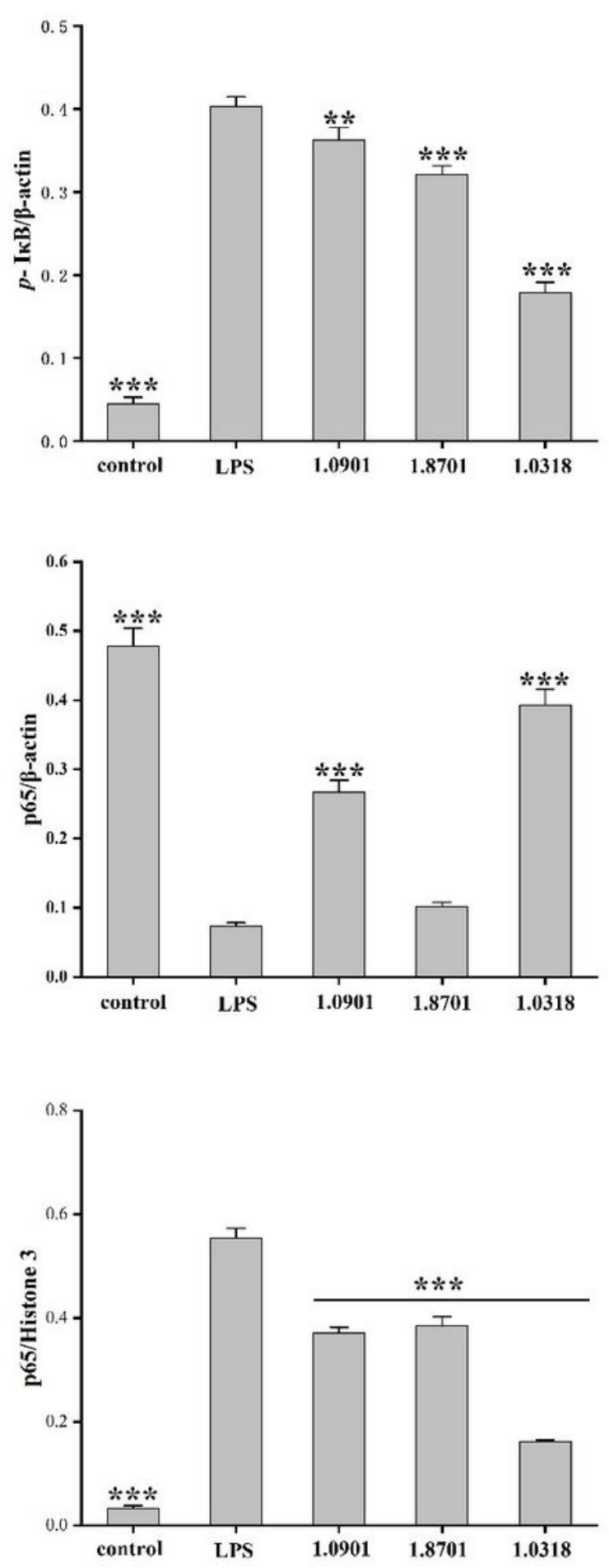

Figure 5 
Effects of the tested strains on LPS-induced NF-KB signaling pathway. Cells were pretreated with L. acidophilus KLDS1.0901, L. helveticus KLDS1.8701 and L. plantarum KLDS1.0318 for $1 \mathrm{~h}$. The IKB, phosphorylation of IKB $₫ A \rrbracket a n d$ nuclear p65, cytoplasm p65 (B) were determined by western blot analysis. Data are expressed as means $\pm S D(n=3) . * P<0.05, * \star P<0.01$ and $* \star \star P<0.001$ vs. the LPS group.

A control LPS 1.09011 .87011 .0318

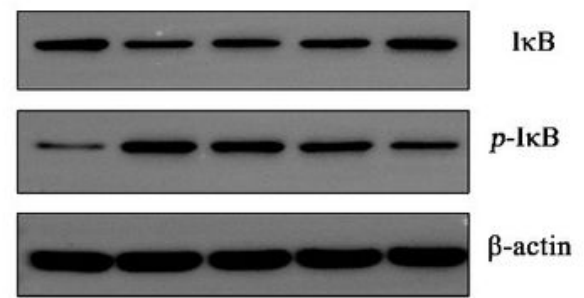

\section{Cytosol}

B

control LPS 1.09011 .87011 .0318
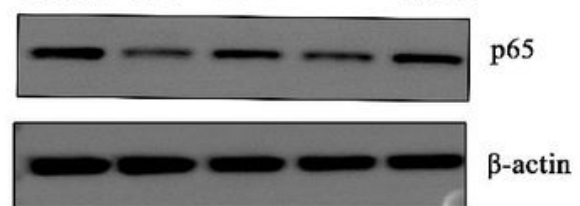

Nucleus

control LPS 1.09011 .87011 .0318
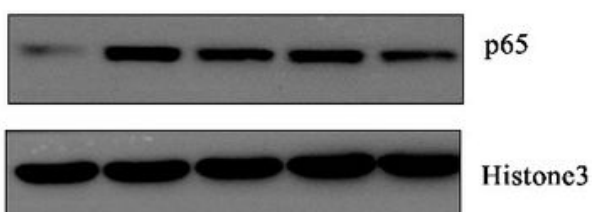
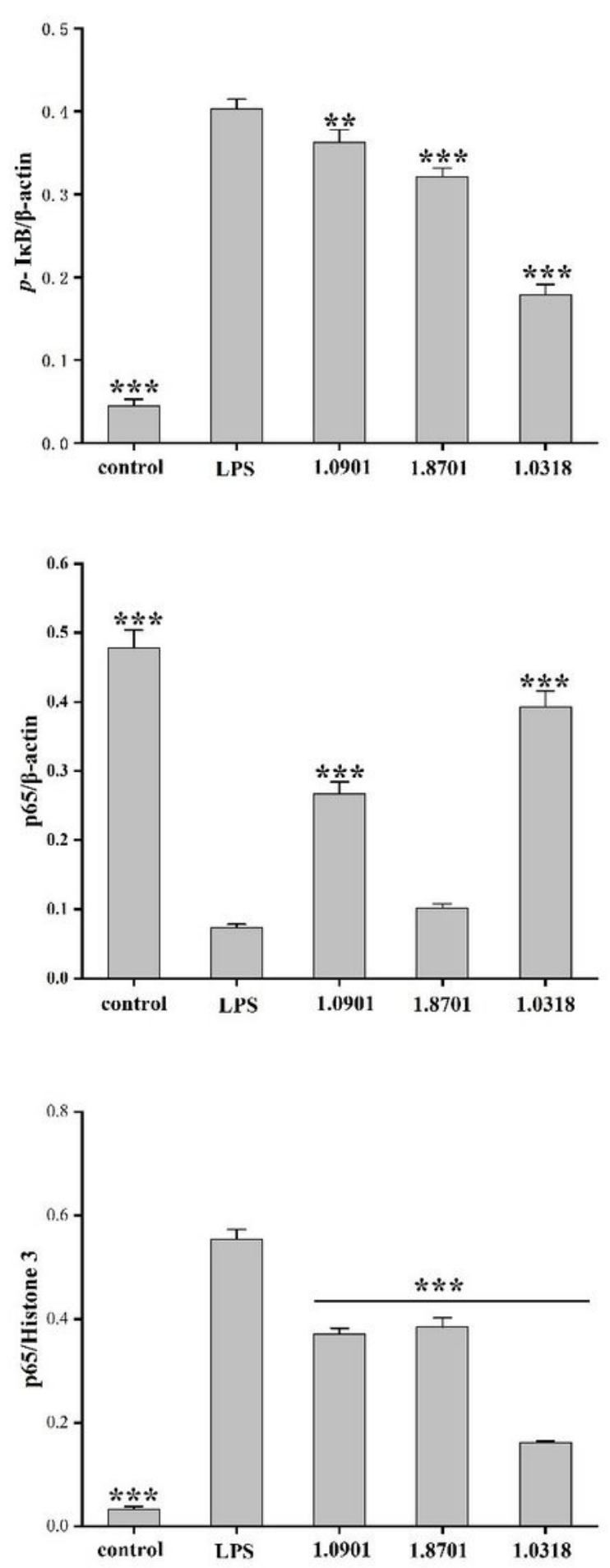

Figure 5 
Effects of the tested strains on LPS-induced NF-KB signaling pathway. Cells were pretreated with L. acidophilus KLDS1.0901, L. helveticus KLDS1.8701 and L. plantarum KLDS1.0318 for $1 \mathrm{~h}$. The IKB, phosphorylation of IKB $₫ A \rrbracket a n d$ nuclear p65, cytoplasm p65 (B) were determined by western blot analysis. Data are expressed as means $\pm S D(n=3) . * P<0.05, * \star P<0.01$ and $* \star \star P<0.001$ vs. the LPS group.
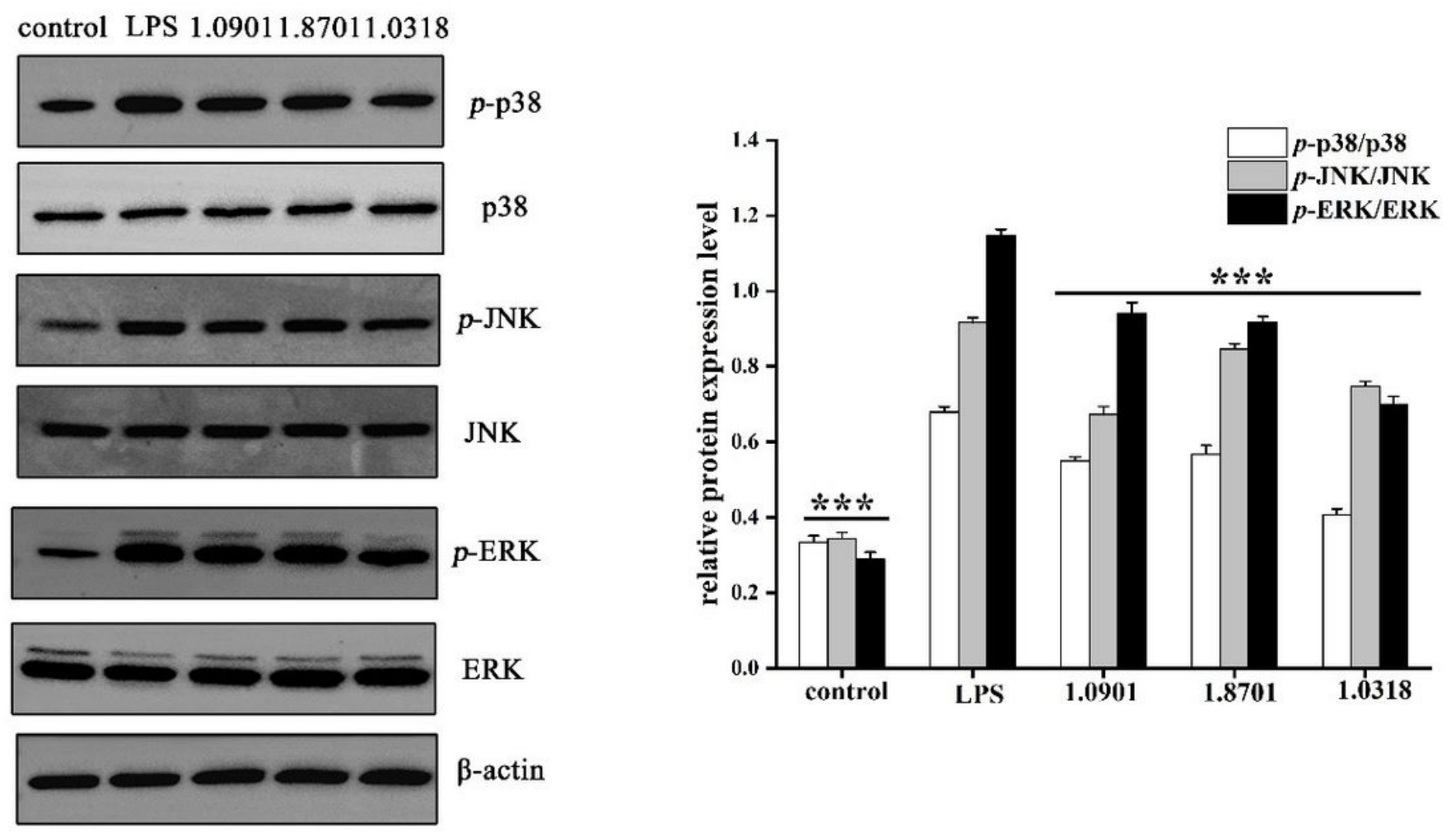

Figure 6

Effects of the tested strains on LPS-induced MAPKs signaling pathway. Cells were pretreated with L. acidophilus KLDS1.0901, L. helveticus KLDS1.8701 and L. plantarum KLDS1.0318 for $1 \mathrm{~h}$. The phosphorylation of p38, ERK and JNK were detected by western blot analysis after LPS stimulated for 20 min. Data are expressed as means $\pm S D(n=3) .{ }^{*} P<0.05$, $* * P<0.01$ and ${ }^{\star * \star} P<0.001$ vs. the LPS group. 
control LPS 1.09011 .87011 .0318
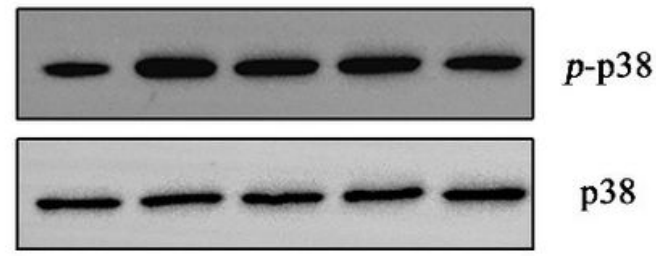

p38
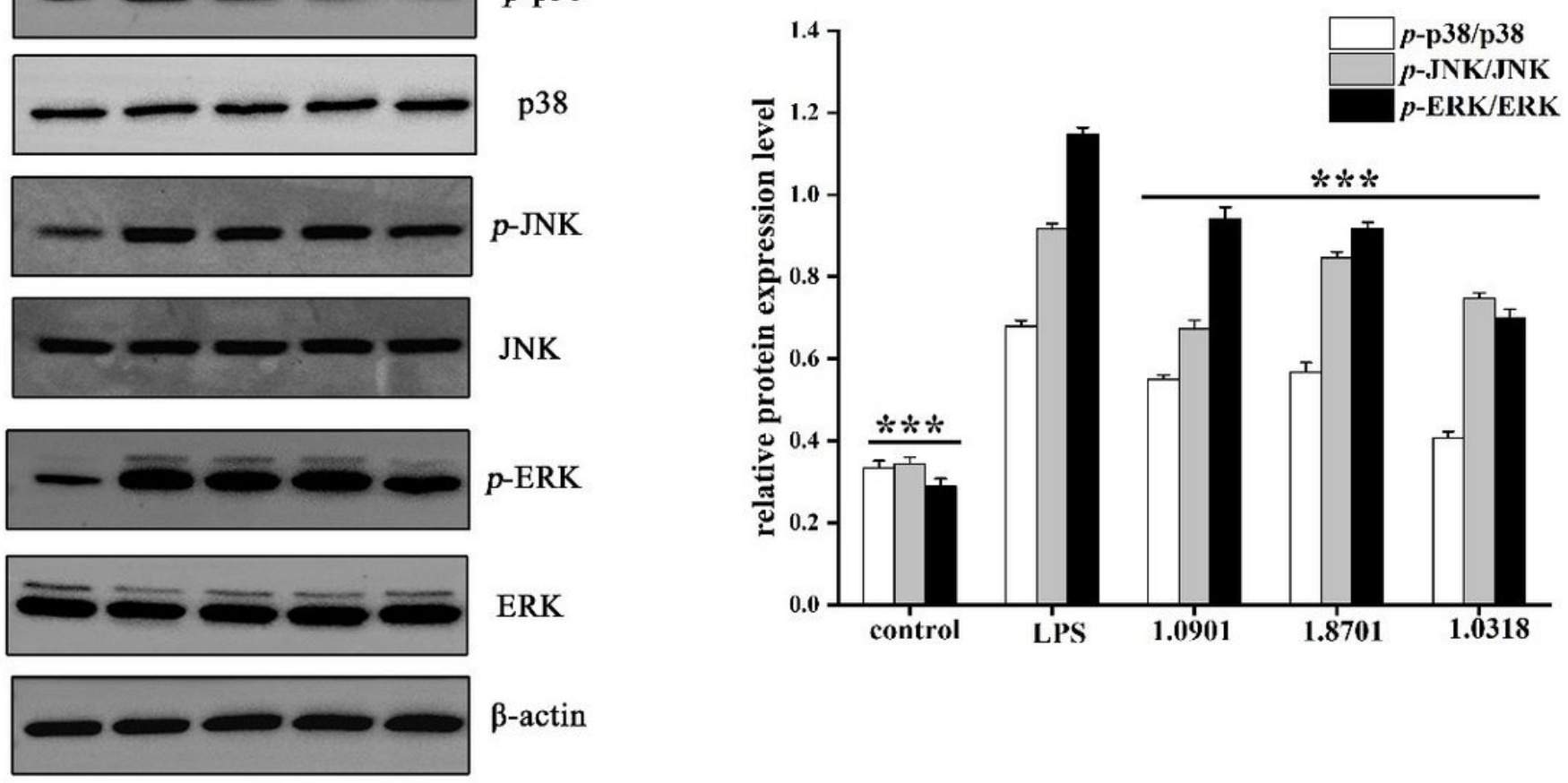

$\beta$-actin

Figure 6

Effects of the tested strains on LPS-induced MAPKs signaling pathway. Cells were pretreated with L. acidophilus KLDS1.0901, L. helveticus KLDS1.8701 and L. plantarum KLDS1.0318 for $1 \mathrm{~h}$. The phosphorylation of p38, ERK and JNK were detected by western blot analysis after LPS stimulated for 20 min. Data are expressed as means $\pm S D(n=3) .{ }^{*} P<0.05$, ${ }^{\star *} P<0.01$ and $* \star * P<0.001$ vs. the LPS group. 


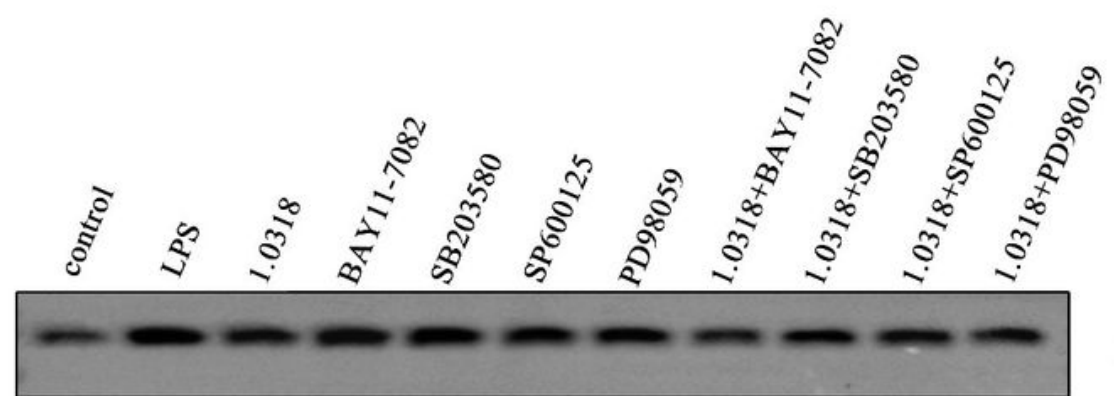

iNOS

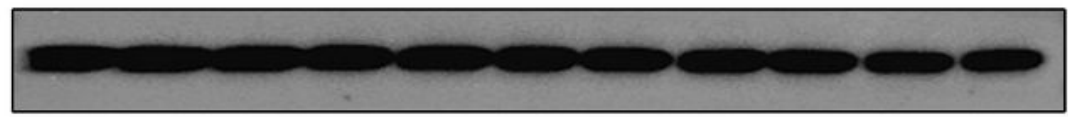

$\beta$-actin

COX-2

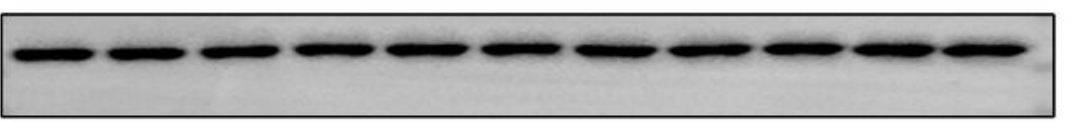

$\beta$-actin

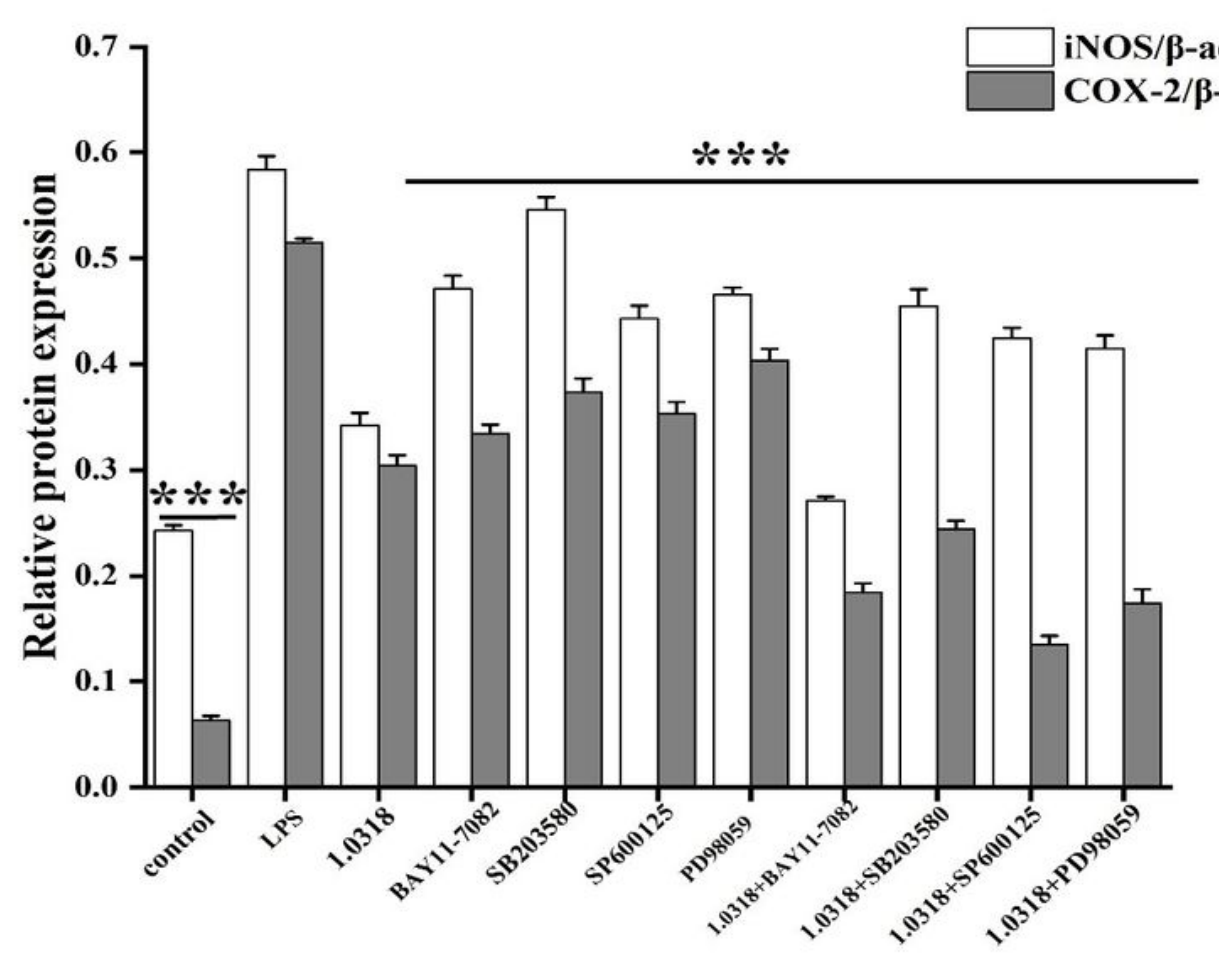

Figure 7

Effects of L. plantarum KLDS1.0318 and NF-KB and MAPKs inhibitors on LPS-induced iNOS and COX-2 protein relative expression in RAW 264.7 cells. Cells were pretreated with three strains for $1 \mathrm{~h}$, iNOS and COX-2 were determined by LPS stimulation for $24 \mathrm{~h}$, respectively. Data are expressed as means \pm SD $(n=$ 3). ${ }^{*} P<0.05$, ** $P<0.01$ and $* \star \star P<0.001$ vs. the LPS group. 


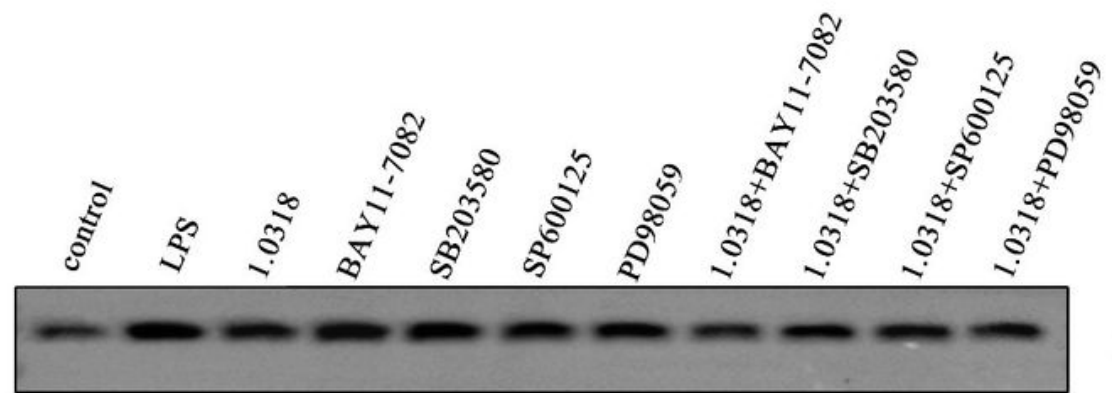

iNOS

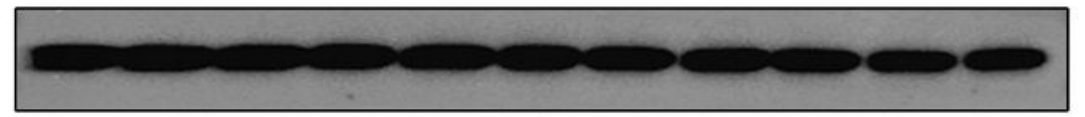

$\beta$-actin

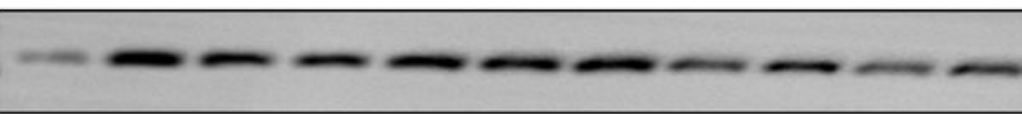

COX-2

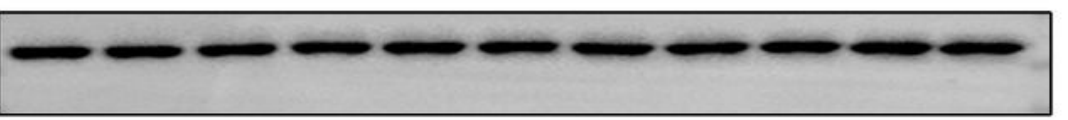

$\beta$-actin

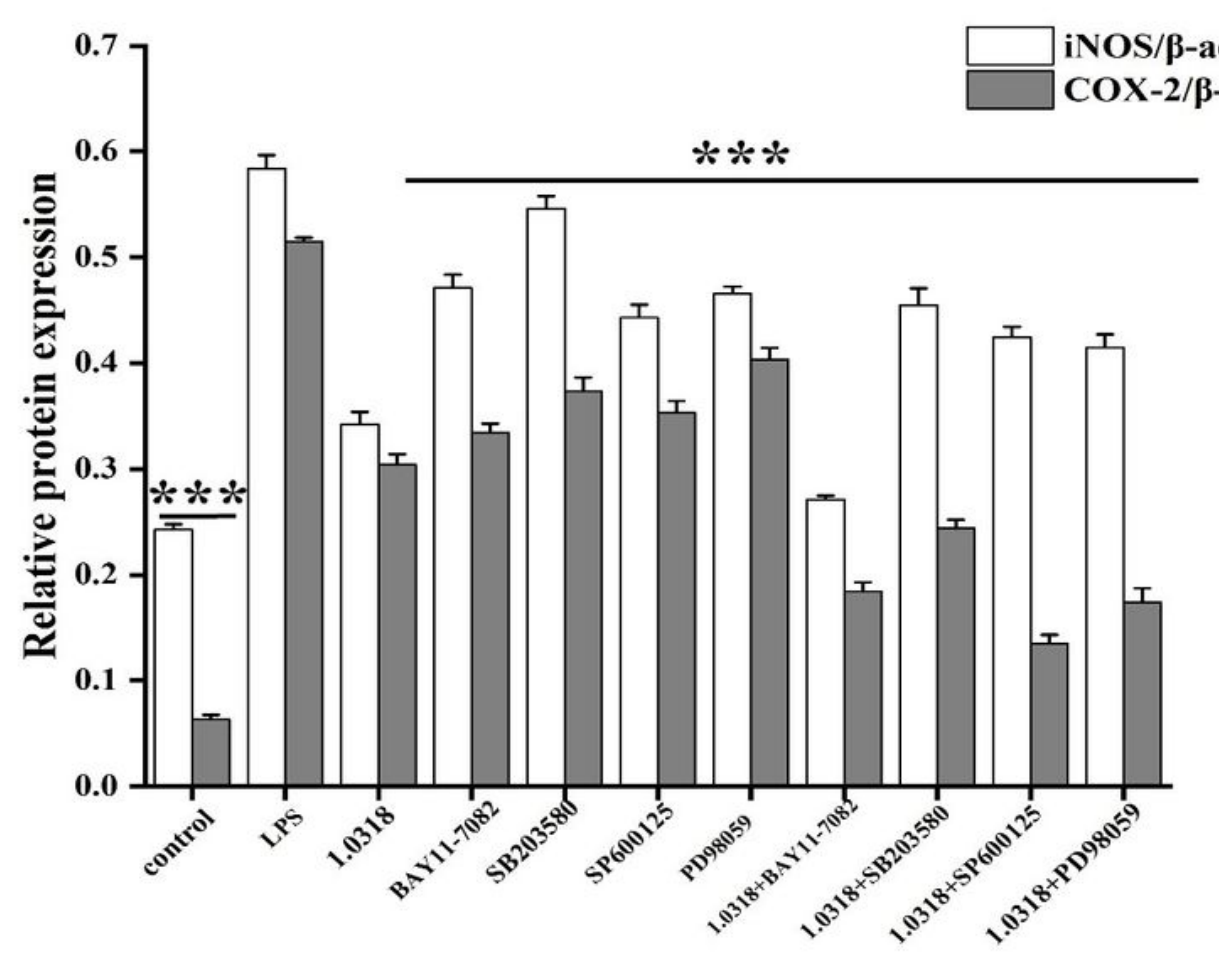

\section{Figure 8}

Effects of L. plantarum KLDS1.0318 and NF-KB and MAPKs inhibitors on LPS-induced iNOS and COX-2 protein relative expression in RAW 264.7 cells. Cells were pretreated with three strains for $1 \mathrm{~h}$, iNOS and COX-2 were determined by LPS stimulation for $24 \mathrm{~h}$, respectively. Data are expressed as means \pm SD $(n=$ 3). * $P<0.05$, ** $P<0.01$ and $* \star \star ~ P<0.001$ vs. the LPS group. 